\title{
1 Common genetic variation influencing human white matter microstructure
}

3 Running title: GWAS of brain white matter

5 Bingxin Zhao ${ }^{1}$, Tengfei $\mathrm{Li}^{2,3}$, Yue Yang ${ }^{1}$, Xifeng Wang ${ }^{1}$, Tianyou Luo ${ }^{1}$, Yue Shan ${ }^{1}$, Ziliang 6 Zhu $^{1}$, Di Xiong ${ }^{1}$, Mads E. Hauberg ${ }^{4,5,7,8}$, Jaroslav Bendl4-6, John F. Fullard ${ }^{4-6}$, Panagiotis

7 Roussos ${ }^{4-6,9}$, Yun Li ${ }^{1,10,11}$, Jason L. Stein ${ }^{10,12}$, and Hongtu Zhu ${ }^{1,3 *}$

$9{ }^{1}$ Department of Biostatistics, University of North Carolina at Chapel Hill, Chapel Hill, NC, USA

$10{ }^{2}$ Department of Radiology, University of North Carolina at Chapel Hill, Chapel Hill, NC, USA

11 BBiomedical Research Imaging Center, School of Medicine, University of North Carolina at Chapel Hill,

12 Chapel Hill, NC, USA

$13{ }^{4}$ Department of Psychiatry, Icahn School of Medicine at Mount Sinai, New York, NY, USA

$14{ }^{5}$ Friedman Brain Institute, Icahn School of Medicine at Mount Sinai, New York, NY, USA

$15{ }^{6}$ Department of Genetics and Genomic Science and Institute for Multiscale Biology, Icahn School of

16 Medicine at Mount Sinai, New York, NY, USA

$177_{\mathrm{iPSYCH}}$, The Lundbeck Foundation Initiative for Integrative Psychiatric Research, Denmark

$18{ }^{8}$ Centre for Integrative Sequencing (iSEQ), Aarhus University, Aarhus, Denmark

$19{ }^{9}$ Mental Illness Research, Education, and Clinical Center (VISN 2 South), James J. Peters VA Medical

20 Center, Bronx, NY, USA

$21{ }^{10}$ Department of Genetics, University of North Carolina at Chapel Hill, Chapel Hill, NC, USA

$22{ }^{11}$ Department of Computer Science, University of North Carolina at Chapel Hill, Chapel Hill, NC, USA

$23{ }^{12}$ UNC Neuroscience Center, University of North Carolina at Chapel Hill, Chapel Hill, NC, USA

$25 \quad{ }^{*}$ Corresponding author:

27 Hongtu Zhu

28 3105C McGavran-Greenberg Hall, 135 Dauer Drive, Chapel Hill, NC 27599.

29 E-mail address: htzhu@email.unc.edu Phone: (919) 966-7250

31 List of Pediatric Imaging, Neurocognition and Genetics (PING) authors provided in the 32 supplemental materials. 


\section{Abstract}

2 Brain regions communicate with each other via tracts of myelinated axons, commonly

3 referred to as white matter. White matter microstructure can be measured in the living

4 human brain using diffusion based magnetic resonance imaging (dMRI), and has been

5 found to be altered in patients with neuropsychiatric disorders. Although under strong

6 genetic control, few genetic variants influencing white matter microstructure have ever

7 been identified. Here we identified common genetic variants influencing white matter

8 microstructure using dMRI in 42,919 individuals $(35,741$ in the UK Biobank). The dMRIs

9 were summarized into 215 white matter microstructure traits, including 105 measures

10 from tract-specific functional principal component analysis. Genome-wide association

11 analysis identified many novel white matter microstructure associated loci $(P<2.3 \times$

$\left.12 \mathrm{10}^{-10}\right)$. We identified shared genetic influences through genetic correlations between

13 white matter tracts and 62 other complex traits, including stroke, neuropsychiatric

14 disorders (e.g., ADHD, bipolar disorder, major depressive disorder, schizophrenia),

15 cognition, neuroticism, chronotype, as well as non-brain traits. Common variants

16 associated with white matter microstructure alter the function of regulatory elements in

17 glial cells, particularly oligodendrocytes. White matter associated genes were enriched

18 in pathways involved in brain disease pathogenesis, neurodevelopment process, and

19 repair of white matter damage $\left(P<1.5 \times 10^{-8}\right)$. In summary, this large-scale tract-specific

20 study provides a big step forward in understanding the genetic architecture of white

21 matter and its genetic links to a wide spectrum of clinical outcomes.

23 Keywords: White Matter Microstructure; dMRI; Diffusion Tensor Imaging; GWAS;

24 Functional Principal Component Analysis; UK Biobank. 
1 Brain functions depend on effective communication across brain regions ${ }^{1}$. White matter

2 comprises roughly half of the human brain and contains most of the brain's long-range

3 communication pathways ${ }^{2}$. White matter tracts build a complex network of structural

4 connections, which keeps the brain globally connected and shapes communication and

5 connectivity patterns ${ }^{3-5}$. Cellular microstructure in white matter tracts plays a pivotal

6 role in maintaining the integrity of connectivity and mediating signal transitions among

7 distributed brain regions ${ }^{6}$. Evidence from neuroscience has further suggested that white

8 matter microstructure may underpin brain function and dysfunction ${ }^{1,7,8}$, and

9 connectivity differences or changes are relevant to a wide variety of neurological and

10 psychiatric disorders, such as attention-deficit/hyperactivity disorder (ADHD) ${ }^{9}$, major

11 depressive disorder $(\mathrm{MDD})^{10}$, schizophrenia ${ }^{11}$, bipolar disorder ${ }^{12}$, multiple sclerosis ${ }^{13}$,

12 Alzheimer's disease ${ }^{14}$, corticobasal degeneration ${ }^{15}$, and Parkinson's disease ${ }^{16}$. White

13 matter microstructural differences and abnormalities can be captured in vivo by

14 diffusion magnetic resonance imaging (dMRI). Using dMRI data, microstructural

15 connectivity can be quantified in diffusion tensor imaging (DTI) models ${ }^{17}$ and measured

16 by several DTI-derived parameters, including fractional anisotropy (FA), mean diffusivity

17 (MD), axial diffusivity (AD), radial diffusivity (RD), and mode of anisotropy (MO). Among

18 them, FA serves as the primary metric of interest in many studies ${ }^{18}$, which is a robust

19 global measure of integrity/directionality and is highly sensitive to general connectivity

20 changes. On the other hand, MD, AD, and RD directly quantify the abstract magnitude of

21 directionalities, and thus are more sensitive to specific types of microstructural

22 changes $^{19}$. In addition, MO can characterize the anisotropy type, describing whether the

23 shape of the diffusion tensor is more linear or planar ${ }^{20,21}$. See Supplementary Note for a

24 global overview of these commonly used DTI parameters.

26 White matter differences in general population cohorts are under strong genetic

27 control. Both family and population-based studies have reported that DTI

28 measurements of white matter microstructure have in general high heritability with

29 estimates varying across different age groups ${ }^{22}$ and tracts $^{23}$. For example, heritability

30 estimates of tract-averaged FA ranged from $53 \%$ to $90 \%$ in twin study of the Human

31 Connectome Project (HCP) ${ }^{24}$. Recent genome-wide association studies (GWAS) of UK

32 Biobank reported an average SNP-based heritability of $48.7 \%$ across different tracts ${ }^{25}$. 
1 Several GWAS $23,25-29$ have been performed to identify loci associated with

2 inter-individual variation in white matter microstructure but shared at least two major

3 limitations: (i) sample size and (ii) spatial specificity. First, the current largest published

4 GWAS of dMRI phenotypes has sample size 17,706 in Zhao, et al. ${ }^{25}$. Similar to other

5 brain-related traits $^{30}$, white matter has a complex and extremely polygenic genetic

6 architecture ${ }^{25,31}$. Large sample size is essential to boost GWAS power in order to identify

7 many common risk variants with small effect sizes. Second, previous GWAS mainly

8 focused on global dMRI measures of the whole brain ${ }^{26,27}$ or tract-averaged (mean)

9 values ${ }^{23,25}$. Global and tract-averaged measures can capture the largest variations in

10 white matter, while reducing the burden to test multiple neuroimaging traits,

11 particularly suitable for GWAS with limited sample size; however, these measures may

12 lose lots of information, as microstructural differences and changes may not have a

13 uniformly consistent pattern across the whole tract. Heterogeneous variation patterns

14 typically exist within voxel-wise DTI maps of the 3D tract curve, which may be more

15 relevant to specific underlying biological processes. For example, previous study found

16 that the association between bipolar disorder and FA is specific to one given segment of

17 the long anterior limb of internal capsule (ALIC) tract connecting prefrontal cortex with

18 the thalamus and brain stem ${ }^{32}$. Due to these limitations, a large number of genetic

19 factors influencing white matter may still be undiscovered. Consequently, with few

20 exceptions (e.g., stroke ${ }^{26}$ and cognitive traits ${ }^{25}$ ), the shared genetic influences between

21 white matter and other complex traits are unknown. Uncovering these potential genetic

22 links may identify important brain regions that are involved in clinical outcomes,

23 especially for brain-related disorders.

25 To overcome these limitations, here we collected individual-level dMRI from five data 26 resources: the UK Biobank ${ }^{33}$, Adolescent Brain Cognitive Development $\left(A B C D^{34}\right), H_{C P}{ }^{35}$,

27 Pediatric Imaging, Neurocognition, and Genetics (PING ${ }^{36}$ ), and Philadelphia

28 Neurodevelopmental Cohort $\left(\mathrm{PNC}^{37}\right)$. We harmonized image processing by using the 29 ENIGMA-DTI pipeline ${ }^{38,39}$ and obtained voxel-wise DTI maps for 42,919 subjects (after 30 quality controls), including 35,741 in UK Biobank. We mainly focused on 21 predefined 31 white matter tracts and generated two groups of phenotypes. The first group contains 32110 tract-averaged parameters for $F A, A D, M D, M O$ and $R D$ in 21 tracts and across the 
1 whole brain. Second, we applied functional principal component analysis $\left(F P C A^{40}\right)$ to

2 generate 105 tract-specific principal components (PCs) for FA by taking the top five PCs

3 of the voxel-wise map within each tract. FPCA is a data-driven approach to characterize

4 the strongest variation components of FA within each tract, which are expected to

5 provide additional microstructural details about axonal organization and myelination

6 omitted by tract-averaged values ${ }^{41,42}$, while limiting multiple testing. More importantly,

7 these PCs may represent FA changes that are more relevant to specific clinical

8 outcomes. We then performed a genome-wide association analysis for these 215

9 phenotypes to discover the genetic architecture of white matter and explore the genetic

10 links to a plethora of clinical endpoints in different trait domains. Our GWAS results

11 have been made publicly available at https://github.com/BIG-S2/GWAS and can be

12 easily browsed through our Brain Imaging Genetics Knowledge Portal (BIG-KP)

13 https://bigkp.web.unc.edu/.

16 RESULTS

17 GWAS Discovery and Validation for 215 DTI parameters.

18 Our discovery analysis utilized data from UKB subjects of British ancestry $(n=33,292)$.

19 All of the $110 \mathrm{DTI}$ mean parameters had significant SNP heritability ${ }^{43}\left(h^{2}\right)$ after

20 Bonferroni adjustment (215 tests, $P<9.4 \times 10^{-31}$, Fig. 1a and Supplementary Table 1).

21 The $h^{2}$ estimates varied from $24.8 \%$ to $65.4 \%$ (mean $h^{2}=46.3 \%$ ), which were

22 comparable with previous results ${ }^{23,25}$. For the 105 tract-specific FA PC parameters, we

23 found that 102 had significant $h^{2}$ (mean $h^{2}=34.1 \%, h^{2}$ range $=(8.6 \%, 65.8 \%), P<1.1 \times$

$\left.2410^{-5}\right)$. The $4^{\text {th }}$ PC of corticospinal tract (CST, 6.2\%), $5^{\text {th }}$ PC of cingulum hippocampus (CGH,

$254.4 \%$ ), and $4^{\text {th }}$ PC of superior fronto-occipital fasciculus (SFO, 3.7\%) had nominally

26 significant $h^{2}$ estimates $(P<0.03)$, which became insignificant after Bonferroni

27 adjustment. The top five PCs in external capsule (EC) were highlighted in bottom panels

28 of Figure 1b. Different from tract-averaged value, these PCs captured more specific FA

29 variations in distinct subfields of EC, all of which had high $h^{2}$ (mean $h^{2}=47.9 \%, h^{2}$ range

$\left.30=(42.9 \%, 52.6 \%), P<1.8 \times 10^{-89}\right)$. Another illustration was given in Supplementary

31 Figure 1 for the PCs of superior longitudinal fasciculus (SLF). These $h^{2}$ results show that

32 the additional microstructural variations captured by unconventional tract-specific FA 
1 PCs are also generally under genetic control. As illustrated in later sections, those

2 heritable local FA variation patterns may also have higher power to identify the shared

3 genetic influences with other complex traits.

5 We performed GWAS for these 215 DTI parameters using 9,023,710 common genetic 6 variants after quality controls (Methods). All Manhattan and QQ plots can be browsed in 7 our BIG-KP server. At a stringent significance level $2.3 \times 10^{-10}$ (i.e., $5 \times 10^{-8} / 215$, 8 additionally adjusted for the 215 phenotypes studied), FUMA ${ }^{44}$ clumped 595 partially 9 independent significant variants (Methods) involved in 1,101 significant associations 10 with 86 FA measures (21 mean and 65 PC parameters, Supplementary Figs. 2-3 and 11 Supplementary Table 2). Genetic variants had broad effects across all white matter 12 tracts, and one variant often influenced multiple FA measures, such as rs12146713 in 13 region 12q23.3, rs309587 in 5q14.3, rs55705857 in 8q24.21, and rs1004763 in 22q13.1. 14 Of the 595 significant variants, 302 were only detected by PC parameters. On average, 15 the number of FA-associated significant variants was 37.0 in each tract (range $=(4,72)$,

16 Fig. 2 and Supplementary Table 3), 50.3\% of which were solely discovered by PC 17 parameters $($ range $=(26.3 \%, 100 \%))$. For example, all of the 22 significant variants 18 associated with CST were detected by PC parameters. Moreover, $66.7 \%(32 / 48)$ of the 19 variants in posterior corona radiata (PCR), 64.9\% (37/57) in posterior thalamic radiation 20 (PTR), 59.7\% (43/72) in SLF, and 56.3\% (18/32) in cingulum cingulate gyrus (CGC) were

21 only associated with PC parameters. These results clearly illustrate the unique 22 contribution of tract-specific PC parameters in identifying genetic variants for FA 23 variations within white matter tract.

25 In addition, 770 significant variants were associated with 83 mean parameters of $A D$, $26 \mathrm{MD}, \mathrm{MO}$ and RD (2,069 significant associations), 565 of these 770 variants (with 967 27 associations) were not identified by FA measures (Fig. 2, Supplementary Figs. 2-3, and 28 Supplementary Table 2). The mean number of significant variants in each tract moved 29 up to 93.3 (range $=(41,160)$ ), and rs13198474 in 6p22.2, rs2267161 in 22q12.2, 30 rs55705857 in $8 q 24.21$, rs7935166 in 11p11.2, and rs7225002 in 7q21.31 were 31 associated with multiple non-FA measures. Of note, more than $70 \%$ of significant 32 variants in cingulum (CGH (90.7\%) and CGC (73.3\%)) were detected by non-FA measures 
1 (Supplementary Table 4), which may suggest that FA is less useful in the thin line-like

2 C-shaped cingulum region than in other tracts. Based on a second and more strict LD

3 clumping (LD $\left.r^{2}<0.1\right)$, FUMA ${ }^{44}$ defined independent lead variants from the above

4 independent significant variants and then genetic loci were characterized (Methods).

5 The 3,170 $(1,101+2,609)$ significant variant-trait associations were summarized as 994

6 significant locus-trait associations (Supplementary Tables 5-6). We then performed

7 functionally informed fine mapping for these locus-level signals using SuSiE $^{45}$ via

8 PolyFun $^{46}$ framework (Methods). PolyFun + SuSiE identified 6,882 variant-trait pairs that

9 had posterior causal probability (i.e., PIP) > 0.95 for 2,299 variants (Supplementary

10 Table 7), suggesting the existence of multiple causal effects in associated loci. In

11 summary, our results illuminate the broad genetics control on white matter

12 microstructural differences. The genetic effects are spread across a large number of

13 variants, consistent with the observed extremely polygenic genetic architecture of many

14 brain-related traits ${ }^{30,47}$.

16 We aimed to find independent replication of our discovery GWAS in five independent

17 validation datasets, all consisting of individuals of European ancestry: the UKB White but

18 Non-British (UKBW, $n=1,809$ ), ABCD European (ABCDE, $n=3,821), \operatorname{HCP}(n=334)$, PING

19 ( $n=461)$, and PNC ( $n=537)$. First, for each DTI parameter, we checked the genetic

20 correlation (gc) between discovery GWAS and the meta-analyzed European validation

21 GWAS (total $n=6,962$ ) by $\operatorname{LDSC}^{48}$ (Methods). The mean gc estimate was 0.95 (standard

22 error $=0.35$ ) across the 215 DTI parameters, 121 of which were significant after

23 adjusting for multiple testing by the Benjamini-Hochberg $(\mathrm{B}-\mathrm{H})$ procedure at 0.05 level

24 (Supplementary Table 8). Genetic correlation estimates near 1 indicates a consistent

25 genetic basis for these phenotypes measured in different cohorts and MRI scanners.

26 Next, we meta-analyzed our discovery GWAS with these European validation GWAS and

27 found that $79.6 \%$ significant associations had smaller $P$-values after meta-analysis,

28 suggesting similar effect size and direction of the top variants in independent

29 cohorts $^{49,50}$. Additionally, we tested for replication by using polygenic risk scores ${ }^{51}$ (PRS)

30 derived from discovery GWAS (Methods). After $\mathrm{B}-\mathrm{H}$ adjustment at 0.05 level $(215 \times 5$

31 tests), the mean number of significant PRS in the five validation GWAS datasets was 195

32 (range $=(193,211), P$ range $=\left(8.5 \times 10^{-27}, 4.5 \times 10^{-2}\right)$, Supplementary Figs. 4-5 and 
1 Supplementary Table 9). Almost all (214/215) DTI parameters had significant PRS in at

2 least one dataset and 165 had significant PRS in all of them, showing the high

3 generalizability of our discovery GWAS results. Across the five validation datasets, the

4 mean additional variance that can be explained by PRS (i.e., incremental R-squared) was

$5 \quad 1.7 \%$ (range $=(0.4 \%, 4.2 \%))$ for the 165 consistently significant DTI parameters. The

6 largest mean (incremental) R-squared was on the $2^{\text {nd }} \mathrm{PC}$ of EC (range $=(2.2 \%, 6.5 \%), P$

7 range $\left.=\left(7.2 \times 10^{-24}, 1.5 \times 10^{-9}\right)\right)$.

9 Finally, we constructed PRS on four non-European validation datasets: the UKB Asian 10 (UKBA, $n=419$ ), UKB Black (UKBBL, $n=211$ ), ABCD Hispanic ( $A B C D H, n=768$ ), and ABCD

11 African American ( $A B C D A, n=1,257)$. The number of significant PRS was 158 and 40 in

12 UKBA and UKBBL, respectively (B-H adjustment at 0.05 level, Supplementary Table 10).

13 In addition, UKBW and UKBA had similar prediction performance (mean $2.38 \%$ vs. $142.33 \%, P=0.67$ ), but the accuracy became significantly smaller in UKBBL (mean $2.38 \%$ 15 vs. $\left.1.67 \%, P=3.9 \times 10^{-9}\right)$. For the two non-European non-UKB datasets, the number of 16 significant PRS was 121 and 114 in $A B C D H$ and $A B C D A$, respectively (B-H adjustment at

170.05 level, Supplementary Table 11), which were much smaller than the ones observed 18 in $A B C D E$. The R-squared were similar between $A B C D H$ and $A B C D E$ (mean $0.74 \%$ vs. $190.69 \%, P=0.28$ ), but the accuracy significantly decreased in ABCDA (mean $0.48 \%$ vs. $\left.200.69 \%, P=1.9 \times 10^{-7}\right)$. These findings show that UKB British GWAS findings have high 21 generalizability in European cohorts, but the generalizability is reduced in 22 cross-population applications, especially in Black/African-American cohorts, highlighting 23 the importance of recruiting sufficient samples from global diverse populations in future 24 genetics discovery of white matter.

\section{Concordance with previous GWAS.}

27 Of the 33,292 subjects in our UKB British discovery GWAS, 17,706 had been used in the 28 largest previous GWAS ${ }^{25}$ for 110 mean parameters. To examine the robustness of their 29 findings, we used the other 15,214 individuals (also removed the relatives ${ }^{52}$ of previous 30 GWAS subjects) to perform a new validation GWAS and then evaluated the strength of 31 replication (Methods). We calculated the replication slope, which was the correlation of 32 the standardized effect size of variants estimated from two independent GWAS ${ }^{53}$. This 
1 analysis was restricted to top $\left(P<1 \times 10^{-6}\right.$ in previous GWAS) independent lead variants

2 after LD-based clumping (window size 250, LD $r^{2}=0.01$ ). The replication slope was 0.84

3 (standard error $\left.=0.02, P<2 \times 10^{-16}\right)$, indicating strong similarity between these top

4 variant effect size estimates. We also applied FINDOR ${ }^{53}$ to reweight $P$-values by

5 leveraging functional enrichments, after which the replication slope increased to 0.86

6 (standard error $\left.=0.02, P<2 \times 10^{-16}\right)$. In addition, for each of the 110 mean parameters,

7 we used $\operatorname{LDSC}^{48}$ to calculate genetic correlation between measurements from the two

8 GWAS. The mean gc estimate was 1.03 (standard error = 0.14, Supplementary Fig. 6 and

9 Supplementary Table 12) across these parameters, all of which were significant after

$10 \mathrm{~B}-\mathrm{H}$ adjustment at 0.05 level $\left(P<1.4 \times 10^{-5}\right)$. In conclusion, these findings indicate that

11 previous UKB GWAS results can be strongly validated in the new UKB British cohort.

13 Next, we carried out association lookups for 1,160 $(595+565)$ independent significant

14 variants (and variants within LD) detected in our UKB British discovery GWAS (Methods).

15 Of the 213 variants (with 696 associations) identified in Zhao, et al. ${ }^{25}, 202$ (with 671

16 associations) were in $\operatorname{LD}\left(r^{2} \geq 0.6\right)$ with our independent significant variants

17 (Supplementary Table 13). On the NHGRI-EBI GWAS catalog 54 , our results tagged many

18 variants that had been implicated with brain structures, including 7 in van der Meer, et

19 al. ${ }^{55}$ for hippocampal subfield volumes, 7 in Verhaaren, et al. ${ }^{56}$ for cerebral white 20 matter hyperintensity (WMH) burden, 5 in Vojinovic, et al. ${ }^{57}$ for lateral ventricular

21 volume, 5 in Rutten-Jacobs, et al. ${ }^{26}$ for WMH and white matter integrity, 2 in Klein, et al.

2258 for intracranial volume, 2 in Hibar, et al. ${ }^{59}$ for subcortical brain region volumes, 2 in

23 Fornage, et al. ${ }^{28}$ for WMH burden, 1 in Elliott, et al. ${ }^{23}$ for brain imaging measurements,

241 in Luo, et al. ${ }^{60}$ for voxel-wise brain imaging measurement, 1 in Hashimoto, et al. ${ }^{61}$ for

25 superior frontal gyrus grey matter volume, 1 in Ikram, et al. ${ }^{62}$ for intracranial volume,

26 and 1 in Sprooten, et al. ${ }^{63}$ for global FA (Supplementary Table 14). When the

27 significance threshold was relaxed to $5 \times 10^{-8}$, we tagged variants reported in more

28 previous studies, such as 2 in Shen, et al. ${ }^{64}$ for brain imaging measurements, 2 in Chung,

29 et al. ${ }^{65}$ for hippocampal volume in dementia, 1 in Chen, et al. ${ }^{66}$ for putamen volume,

30 and 1 in Christopher, et al. ${ }^{67}$ for posterior cingulate cortex (Supplementary Table 15).

31 For example, we observed colocalizations in region 5q14.3 with previously reported

32 variants for $\mathrm{WMH}$ volume and white matter integrity ${ }^{26}$, in $10 q 26.13$ with hippocampal 
1 volumes $^{55}$, in $17 q 21.31$ with subcortical ${ }^{59}$ and intracranial ${ }^{62}$ volumes, and in $17 q 25.1$

2 with WMH volume $26 /$ burden 28,56 (Supplementary Fig. 7).

4 Moreover, we found lots of previous associations with other complex traits in different 5 domains (Supplementary Table 16). We highlighted 190 variants with psychological 6 traits (e.g., neuroticism ${ }^{68}$, well-being spectrum ${ }^{69}$, general risk tolerance ${ }^{70}$ ), 179 with

7 cognitive/educational traits (e.g., cognitive ability ${ }^{71}$, educational attainment ${ }^{72}$ ), 99 with

8 psychiatric disorders (e.g., schizophrenia ${ }^{73}, \mathrm{MDD}^{74}$, bipolar disorder ${ }^{75}, \mathrm{ADHD}^{76}$, autism 9 spectrum disorder ${ }^{77}$ ), 95 with anthropometric traits (e.g., height ${ }^{78}$, body mass index $\left.10(\mathrm{BMI})^{53}\right), 68$ with bone mineral density ${ }^{79,80}, 54$ with smoking/drinking (e.g., smoking ${ }^{81}$, 11 alcohol use disorder ${ }^{82}$ ), 20 with neurological disorders (e.g., corticobasal degeneration ${ }^{83}$, 12 Parkinson's disease ${ }^{84}$, Alzheimer's disease $\mathrm{e}^{85}$, multiple sclerosis ${ }^{86}$ ), 18 with sleep (e.g., 13 sleep duration ${ }^{87}$, chronotype ${ }^{88}$ ), 11 with glioma (glioblastoma or non-glioblastoma) 14 tumors $^{89,90}$, and 6 with stroke ${ }^{91-93}$. For example, white matter associated variants 15 colocalized with many risk variants of cognitive/educational traits as well as 16 brain-related disorders in regions 17q21.31, 6p22.1, and 6p22.2 (Supplementary Fig. 8).

17 Strong colocalizations were also found in 7p22.3 with anthropometric traits and bone 18 mineral density, in 10p12.31 with smoking/drinking and anthropometric traits, in 9p21.3 19 with glioma and stroke, and in 8q24.12 with bone mineral density (Supplementary Fig. 20 9).

22 To further explore these overlaps, we summarized the number of previously reported 23 variants of other traits that can be tagged by any DTI parameters in each white matter 24 tract (Supplementary Table 17). We found that variants associated with psychological, 25 cognitive/educational, smoking/drinking traits and neurological and psychiatric 26 disorders were globally linked to many white matter tracts (Supplementary Fig. 10). For 27 traits in other domains, the overlaps may have some tract-specific patterns. For 28 example, 3 of the 6 variants associated with stroke were linked to both SFO and ALIC, 29 and the other 3 were found in superior corona radiata (SCR), anterior corona radiata 30 (ACR), genu of corpus callosum (GCC), body of corpus callosum (BCC), EC, posterior limb 31 of internal capsule (PLIC), and posterior limb of internal capsule (RLIC). In addition, 7 of 32 the 11 risk variants of glioma were associated with splenium of corpus callosum (SCC), 
112 of the 18 variants reported for sleep were related to PLIC or inferior fronto-occipital

2 fasciculus (IFO), and 26 of the 68 variants associated with bone mineral density were

3 linked to CST. In addition, more than half of the variants tagged by uncinate fasciculus

4 (UNC) and fornix (FX) had been implicated with anthropometric traits. We carried out

5 voxel-wise association analysis for four representative pleiotropic variants (Methods).

6 Figure 3 illustrated their genomic locations and voxel-wise effect size patterns in spatial

7 brain maps. rs593720 and rs13198474 had strong effects in corpus callosum (GCC, BCC,

8 and SCC), corona radiata ( $A C R$ and SCR), and EX, and the two variants widely tagged

9 psychiatric $^{94}$ and neurological ${ }^{95}$ disorders, as well as psychological ${ }^{96}$ and

10 cognitive/educational ${ }^{97}$ traits. On the other hand, rs77126132 highlighted in SCC and

11 BCC was particularly linked to glioma ${ }^{89}$, and rs798510 in SCR, FX, and PLIC was

12 associated with several anthropometric traits ${ }^{98}$.

14 An atlas of genetic correlations with other complex traits.

15 Because of the shared loci associated with both white matter microstructure and other

16 complex traits, we systematically examined their pairwise genetic correlations by using

17 our discovery GWAS summary statistics $(n=33,292)$ and publicly available

18 summary-level data of other 76 complex traits via LDSC (Methods, Supplementary Table

19 18). There were 760 significant pairs between 60 complex traits and 175 DTI parameters

20 after $\mathrm{B}-\mathrm{H}$ adjustment at 0.05 level $\left(76 \times 215\right.$ tests, $P$ range $=\left(8.6 \times 10^{-12}, 2.3 \times 10^{-3}\right)$,

21 Supplementary Table 19), 38.3\% (291/760) of which were detected by PC parameters.

22 We found that DTI parameters were widely correlated with subcortical and WMH

23 volumes (Supplementary Fig. 11), brain-related traits (Supplementary Fig. 12), and

24 other non-brain traits (Supplementary Fig. 13). To validate these results, we performed

25 cross-trait PRS separately on our five European validation GWAS datasets and LDSC on

26 their meta-analyzed summary statistics ( $n=6,962$, Methods). We found that 681

27 (89.6\%) of these 760 significant pairs can be validated in at least one of the six validation

28 analyses after $\mathrm{B}-\mathrm{H}$ adjustment at 0.05 level (760 tests, $P$ range $=\left(1.7 \times 10^{-10}, 2.9 \times 10^{-2}\right)$,

29 Supplementary Table 20), indicating the robustness of our findings. We then reran LDSC

30 after meta-analyzed our UKB British discovery GWAS with these European validation

31 GWAS ( $n=40,254$ ). The number of significant pairs increased to 855 between 62 
1 complex traits and 178 DTI parameters (Fig. 4, Supplementary Figs. 14-16 and

2 Supplementary Table 21).

4 We replicated previously reported genetic correlations with cognitive/educational 5 traits ${ }^{25}$, drinking behavior ${ }^{25}$, stroke ${ }^{23,26}$, and $\mathrm{MDD}^{25,26}$, and more tract-specific details 6 were revealed. For example, stroke (any subtypes) and ischemic stroke subtypes ${ }^{92}$ (large 7 artery stroke, cardioembolic stroke, and small vessel stroke) showed broad genetic 8 correlations with corpus callosum ( $G C C$ and $B C C$ ), corona radiata (ACR, SCR, and PCR), 9 limb of internal capsule (PLIC, ALIC), EC, SLF, SFO, and UNC $(|g C|$ range $=(0.16,0.42), P<$ $\left.102.5 \times 10^{-3}\right)$, matching findings in our association lookups. We further observed that small 11 vessel stroke subtype had specific but higher genetic correlations with ALIC and SFO $12\left(|\mathrm{gc}|\right.$ range $\left.=(0.52,0.69), P<1.2 \times 10^{-3}\right)$. In contrast, there were no significant genetic 13 correlations detected for large artery and cardioembolic stroke, demonstrating the 14 potentially much stronger genetic links between white matter tracts and small vessel 15 stroke subtype.

17 More importantly, many new genetic correlations were uncovered for brain-related 18 traits, such as Alzheimer's disease, ADHD, bipolar disorder, schizophrenia, chronotype, 19 insomnia, neuroticism, and risk tolerance. For example, significant genetic correlation 20 was found between PTR and Alzheimer's disease $\left(|\mathrm{gc}|=0.30, P=1.7 \times 10^{-3}\right)$, EC and $21 \operatorname{ADHD}\left(|\mathrm{gc}|=0.18, P=4.5 \times 10^{-5}\right)$, UNC and bipolar disorder $\left(|\mathrm{gc}|>0.15, P<4.0 \times 10^{-4}\right)$,

22 and SLF and schizophrenia $\left(|\mathrm{gc}|=0.11, P=2.3 \times 10^{-3}\right)$, matching previously reported 23 case-control differences ${ }^{12,99-101}$ on these tracts. We also found novel significant 24 correlations for non-brain traits, including high blood pressure, height, BMI, bone 25 mineral density, number of non-cancer illnesses and treatments, heavy manual or 26 physical work, smoking, coronary artery disease, lung function, and type 2 diabetes

27 (T2D). For example, high blood pressure was genetically correlated with 19 tracts 28 including SFO, SLF, UNC, EC, and ALIC $\left(\mid\right.$ gc $\mid$ range $\left.=(0.09,0.25), P<2.4 \times 10^{-3}\right)$. Previous 29 research found widespread associations between human brain and these traits, such as 30 bone mineral density ${ }^{102}$, hypertension ${ }^{103}$, T2D $^{104}$, lung function ${ }^{105}$, heart disease ${ }^{106}$, and 31 anthropometric traits ${ }^{107}$. Our findings further illuminate their underlying genetic links.

32 We summarized significant genetic correlations identified in each tract and found that 
$132.3 \%$ (120/372) of these tract-trait genetic correlations can only be detected by PC

2 parameters (Supplementary Fig. 17 and Supplementary Table 22). For example, most of

3 the significant genetic correlations in EC were solely detected by its PC parameters, such

4 as $\mathrm{ADHD}, \mathrm{BMI}$, cognitive function, neuroticism, and insomnia.

6 We explored partial genetic causality among these traits using the latent causal 7 variable ${ }^{108}$ (LCV) model (Methods). As suggested, we conservatively restricted the LCV 8 analysis to pairs with at least nominally significant genetic correlation $(P<0.05)$, 9 significant evidence of genetic causality (B-H adjustment at 0.01 level, $76 \times 215$ tests), 10 and large genetic causality proportion estimate $(|\mathrm{GCP}|>0.6)$, which were extremely 11 unlikely to be false positives ${ }^{108}$. The LCV model suggested that high blood pressure was 12 partially genetically causal for white matter $\left(|\mathrm{GCP}|>0.67, P<2.2 \times 10^{-5}\right.$,

13 Supplementary Fig. 18 and Supplementary Table 23). On the other hand, white matter 14 may have partially genetically causal effects on insomnia, under sleep, and neuroticism $15\left(|\mathrm{GCP}|>0.64, P<7.1 \times 10^{-8}\right)$. These findings may lead to plausible biological hypotheses 16 in future research and suggest the existence of different biological mechanisms 17 underlying the atlas of genetic correlations. More efforts are required to explore causal 18 relationships and the shared biological processes ${ }^{109}$ among these genetically correlated 19 traits.

21 Gene-level analysis.

22 We carried out MAGMA ${ }^{110}$ gene-based association analysis for the 215 DTI parameters 23 using our discovery GWAS summary statistics (Methods). There were 3,903 significant 24 gene-level associations ( $P<1.2 \times 10^{-8}$, adjusted for 215 phenotypes) between 620 genes 25 and 179 DTI parameters (Supplementary Table 24), 153 of the associated genes can 26 only be discovered by PC parameters. We replicated 99 of 112 MAGMA genes reported 27 in Zhao, et al. ${ }^{25}, 8$ white matter-associated genes (SH3PXD2A, NBEAL1, C1QL1, COL4A2, 28 TRIM47, TRIM65, UNC13D, FBF1) in Verhaaren, et al. 56, 4 (VCAN, TRIM47, XRCC4, 29 HAPLN1) in Rutten-Jacobs, et al. ${ }^{26}, 3$ (ALDH2, PLEKHG1, TRIM65) in Traylor, et al. ${ }^{27}, 3$ 30 (ALDH2, PLEKHG1, TRIM65) in Hofer, et al. ${ }^{111}, 2$ (TRIM47, TRIM65) in Fornage, et al. ${ }^{28}$, 31 and 2 (GNA12, GNA13) in Sprooten, et al. ${ }^{112}$. Most of the other genes had not been 32 implicated with white matter. Many of our MAGMA genes had been linked to other 
1 complex traits (Supplementary Table 25), such as 70 genes in Anney, et al. ${ }^{94}$ for autism

2 spectrum disorder or schizophrenia, 50 in Morris, et al. ${ }^{79}$ for heel bone mineral density,

338 in Hoffmann, et al. ${ }^{113}$ for blood pressure variation, 51 in Linnér, et al. ${ }^{70}$ for risk

4 tolerance, 36 in Rask-Andersen, et al. ${ }^{98}$ for body fat distribution, and 26 in Hill, et al. ${ }^{114}$

5 for neuroticism.

7 Next, we mapped significant variants $\left(P<2.3 \times 10^{-10}\right)$ to genes according to physical 8 position, expression quantitative trait loci (eQTL) association, and 3D chromatin (Hi-C) 9 interaction via FUMA ${ }^{44}$ (Methods). FUMA yielded 1,189 new associated genes (1,630 in 10 total) that were not discovered in MAGMA analysis (Supplementary Table 26), 11 replicating 286 of the 292 FUMA genes identified in Zhao, et al. ${ }^{25}$ and more other genes 12 in previous studies of white matter, such as $P D C D 11^{56}, A C O X 1^{56}, C L D N 23^{111}$, 13 EFEMP $1^{26,27,56}$, and IRS2 $2^{111}$. More overlapped genes were also observed between white 14 matter and other traits (Supplementary Table 27). Particularly, 876 FUMA genes were 15 solely mapped by significant Hi-C interactions in brain tissues (Supplementary Table 28), 16 demonstrating the power of integrating chromatin interaction profiles in GWAS of white 17 matter.

19 We then explored the gene-level pleiotropy between white matter and 79 complex 20 traits, including nine neurological and psychiatric disorders ${ }^{115}$ studied in Sey, et al. ${ }^{115}$

21 and (other) traits studied in our genetic correlation analysis. For brain-related traits, the 22 associated genes were predicted by the recently developed Hi-C-coupled MAGMA ${ }^{115}$ 23 (H-MAGMA) tool (Methods). Traditional MAGMA ${ }^{110}$ was used for non-brain GWAS. 24 H-MAGMA prioritized 737 significant genes for white matter $\left(P<6.3 \times 10^{-9}\right.$, adjusted for 25215 phenotypes and two brain tissue types, Supplementary Table 29), and we focused 26 on 329 genes that can be replicated in our meta-analyzed European validation GWAS ( $n$ $27=6,962)$ at nominal significance level $(P<0.05$, Supplementary Table 30). We found 28 that 298 of these 329 genes were associated with at least one of 57 complex traits 29 (Supplementary Table 31). Supplementary Figure 19 and Supplementary Table 32 30 display the number of overlapped genes between 57 complex traits and 21 white matter 31 tracts. Most white matter tracts have many pleiotropic genes with other complex traits, 32 aligning with patterns in association lookups and genetic correlation analysis. For 
1 example, schizophrenia had 80 overlapped genes with SLF, 71 with CGC, 68 with EC, and

265 with SCR. Global white matter changes in schizophrenia patients had been

3 observed $^{101,116,117}$. Particularly, 230 white matter H-MAGMA genes had been identified

4 in Sey, et al. ${ }^{115}$ for nine neurological and psychiatric disorders (Supplementary Table

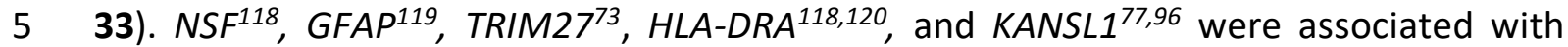

6 five of these disorders, and another 69 genes were linked to at least three different

7 disorders (Supplementary Fig. 20). In summary, our analysis largely expands the

8 overview of gene-level pleiotropy, informing the shared genetic influences between

9 white matter and other complex traits.

11 Biological annotations.

12 In order to identify tissues and cell types where genetic variation leads to changes in

13 white matter microstructure, we performed partitioned heritability analyses ${ }^{121}$ from the

14 GWAS of global FA and MD within tissue type and cell type specific regulatory elements.

15 First, we utilized regulatory elements across multiple adult and fetal tissues ${ }^{122}$. As

16 expected, both FA and MD had the most significant enrichment of heritability in active

17 gene regulation regions of brain tissues (Fig. 5a, Supplementary Fig. 21, and

18 Supplementary Table 34). To identify gross cell types, we again performed partitioned

19 heritability using chromatin accessibility data of two brain cell types, neurons (NeuN+)

20 and glia (NeuN-) sampled from 14 brain regions, including both cortical and

21 subcortical ${ }^{123}$. For all regions, we found that significant enrichment of FA and MD

22 heritability existed in glial but not neuronal regulatory elements after B-H adjustment at

230.05 level (Fig. 5b). These results are expected as white matter is largely composed of

24 glial cell types. For further resolution on cell types, we tested partitioned heritability

25 enrichment within differentially accessible chromatin of glial cell subtypes,

26 oligodendrocyte (NeuN-/Sox10+), microglia and astrocyte (NeuN-/Sox10-) and two

27 neuronal cell subtypes GABAergic (NeuN+/Sox6+) and glutamatergic neurons

28 (NeuN+/Sox6-) (Methods). Heritability of FA and MD was significantly enriched in

29 oligodendrocyte, microglia, and astrocyte annotations $\left(P<4.8 \times 10^{-3}\right)$. The

30 oligodendrocyte annotation accounted for $10.4 \%$ (standard error $=2.6 \%, P=9.5 \times 10^{-5}$ )

31 of the FA heritability while only composed $0.3 \%$ of the variants. In contrast, no

32 significant enrichment was observed in neurons (Fig. 5c). These analyses imply that 
1 common variants associated with white matter microstructure alter the function of

2 regulatory elements in glial cells, particularly oligodendrocytes, the cell type expected to

3 influence white matter microstructure, providing strong support of the biological

4 validity of the genetic associations.

6 To gain more insights into biological mechanisms, we performed several analyses to

7 explore biological interpretations of white matter associated genes. First, MAGMA gene

8 property $^{110}$ analysis was carried out for $13 \mathrm{GTEx}^{124}$ (v8) brain tissues to examine whether

9 the tissue-specific gene expression levels were related to significance between genes

10 and DTI parameters (Methods). After Bonferroni adjustment (13 $\times 215$ tests), we

11 detected 57 significant associations for gene expression in brain cerebellar hemisphere

12 and cerebellum tissues $\left(P<1.8 \times 10^{-5}\right.$, Supplementary Fig. 22 and Supplementary Table

13 35), suggesting that genes with higher transcription levels on white matter-presented

14 regions also had stronger genetic associations with DTI parameters. In contrast, no

15 signals were observed on regions primarily dominated by grey matter, such as basal

16 ganglia and cortex. Next, we performed drug target lookups in a recently established

17 drug target network ${ }^{125}$, which included 273 nervous system drugs (ATC code starts with

18 " $N$ ") and 241 targeted genes. We found that 19 white matter associated genes were

19 targets for 104 drugs, 43 of which were anti-psychotics (ATC: N05A, target such as

$20 D R D 4)$ to manage psychosis like schizophrenia and bipolar, 40 were anti-depressants

21 (ATC: N06A, target such as SLC6A4) to treat MDD and other conditions, 14 were

22 anti-Parkinson drugs (ATC: N04B, target such as HTR2B), and 14 were anti-convulsants

23 (ATC: N03A, target such as SCN5A) used in the treatment of epileptic seizures

24 (Supplementary Table 36). In addition, we treated white matter associated genes as an

25 annotation and performed partitioned heritability enrichment analysis ${ }^{121}$ for the other

2676 complex traits (Methods). After B-H adjustment at 0.05 level, heritability of 54

27 complex traits was significantly enriched in regions influencing DTI parameters

28 (Supplementary Fig. 23 and Supplementary Table 37). These results suggest the

29 potential clinical values of the genes identified for white matter microstructure.

31 MAGMA $^{110}$ competitive gene-set analysis was performed for 15,496 gene sets $(5,500$

32 curated gene sets and 9,996 GO terms, Methods). We found 180 significant gene sets 
1 after Bonferroni adjustment $\left(15,496 \times 215\right.$ tests, $P<1.5 \times 10^{-8}$, Supplementary Table 38).

2 The top five frequently prioritized gene sets were "dacosta uv response via ercc $3 d_{n}$ "

3 (M4500), "dacosta uv response via ercc3 common dn" (M13522), "graessmann

4 apoptosis by doxorubicin $\mathrm{dn}^{\prime}$ (M1105), "gobert oligodendrocyte differentiation $\mathrm{dn}^{\prime}$

5 (M2369), and "blalock alzheimers disease up" (M12921). M4500 and M13522 are

6 ERCC3-associated gene sets related to xeroderma pigmentosum (XP) and

7 trichothiodystrophy (TTD) syndromes, which are genetic disorders caused by a defective

8 nucleotide excision repair system ${ }^{126,127}$. In addition to skin symptoms, patients of XP and

9 TTD often reported various neurological deteriorations and white matter abnormalities,

10 such as intellectual impairment ${ }^{128}$, myelin structures degradation ${ }^{129}$, and diffuse

11 dysmyelination ${ }^{130}$. M1105 regulates the apoptosis of breast cancer cells in response to

12 doxorubicin treatment. Clinical research found that breast cancer chemotherapy like

13 doxorubicin was neurotoxic ${ }^{131}$ and can cause therapy-induced brain structural changes

14 and decline in white matter integrity ${ }^{132}$. M2369 plays a critical role in oligodendrocyte

15 differentiation, which mediates the repair of white matter after damaging events ${ }^{133}$, and

16 M12921 is related to the pathogenesis of Alzheimer's disease ${ }^{134}$.

18 Several gene sets of rat sarcoma (Ras) proteins, small GTPases, and rho family GTPases

19 were also prioritized by MAGMA, such as "go regulation of small gtpase mediated signal

20 transduction" (GO: 0051056), "go small gtpase mediated signal transduction" (GO:

21 0007264), "go re gelation of ras protein signal transduction" (GO: 0046578), "go ras

22 protein signal transduction" (GO: 0007265), and "reactome signaling by rho gtpases"

23 (M501). Ras proteins activity is involved in developmental processes and abnormalities

24 of neural cells in central nervous system ${ }^{135,136}$; small and rho family GTPases play crucial

25 roles in basic cellular processes during the entire neurodevelopment process and are

26 closely connected to several neurological disorders ${ }^{137-139}$. We also observed significant

27 enrichment in pathways related to nervous system, including "go neurogenesis" (GO:

28 0022008), "go neuron differentiation" (GO: 0030182), "go neuron development" (GO:

29 0048666), "go regulation of neuron differentiation" (GO: 0045664), and "go regulation

30 of nervous system development" (GO: 0051960). Finally, we applied DEPICT ${ }^{140}$ gene-set

31 enrichment testing for 10,968 pre-constituted gene sets (Methods), 7 of which survived

32 Bonferroni adjustment $\left(10,968 \times 215\right.$ tests, $\left.P<2.1 \times 10^{-8}\right)$, such as two gene sets 
1 involved in Ras proteins and small GTPases (GO: 0046578 and GO: 0005083) and

2 another two for vasculature and blood vessel developments (GO: 0001944 and GO:

3 0001568, Supplementary Table 39). More MAGMA enriched gene sets can also be

4 detected by DEPICT when the significance threshold was relaxed to $6.5 \times 10^{-6}$ (i.e., not

5 adjusted for testing 215 phenotypes). In summary, our results provide many insights

6 into the underlying biological processes of white matter, suggesting that DTI measures

7 could be useful in understanding the shared pathophysiological pathways between

8 white matter microstructure and multiple diseases and disorders.

\section{DISCUSSION}

11 In this study, we analyzed the genetic architecture of brain white matter using dMRI

12 scans of 42,919 subjects collected from five publicly accessible data resources. Through

13 a genome-wide analysis, we identified hundreds of previously unknown variants and

14 genes for white matter microstructural differences. Many previously reported genetic

15 hits were confirmed in our discovery GWAS, and we further validated our discovery

16 GWAS in a few replication cohorts. We evaluated the genetic relationships between

17 white matter and a wide variety of complex traits in association lookups, genetic

18 correlation estimation, and gene-level analysis. A large proportion of our findings were

19 revealed by unconventional tract-specific PC parameters. Bioinformatics analyses found

20 tissue and cell-specific functional enrichments and lots of enriched biological pathways.

21 Together, these results suggest the value of large-scale neuroimaging data integration

22 and the application of tract-specific FPCA in studying the genetics of human brain.

24 One limitation of the present study is that the majority of publicly available dMRI data

25 are from subjects of European ancestry and our discovery GWAS focused on UKB British

26 individuals. Such GWAS strategy can efficiently avoid false discoveries due to population

27 stratifications and heterogeneities across studies ${ }^{23,141}$, but may raise the question that

28 to what degree the research findings can be generalized and applied to global

29 populations ${ }^{142,143}$. In our analysis, we found that the UKB British-derived PRS were still

30 widely significant in Hispanic, Asian, and Black/African American testing cohorts but had

31 reduced performances, especially in Black/African American cohorts. This may indicate

32 that the genetic architecture of white matter is similar but not the same across different 
1 populations. Identifying the cross-population and population-specific components of

2 genetic factors for human brain could be an interesting future topic. As more

3 non-European neuroimaging data become available (e.g., the ongoing CHIMGEN

4 project $^{144}$ in Chinese population), global integration efforts are needed to study the

5 comparative genetic architectures and to explore the multi-ethnic genetics relationships

6 among brain and other human complex traits.

8 URLS.

9 Brain Imaging GWAS Summary Statistics, https://github.com/BIG-S2/GWAS;

10 Brain Imaging Genetics Knowledge Portal, https://bigkp.web.unc.edu/;

11 UKB Imaging Pipeline, https://git.fmrib.ox.ac.uk/falmagro/UK biobank pipeline v 1;

12 ENIGMA-DTI Pipeline, http://enigma.ini.usc.edu/protocols/dti-protocols/;

13 PLINK, https://www.cog-genomics.org/plink2/;

14 GCTA \& fastGWA, http://cnsgenomics.com/software/gcta/;

15 METAL, https://genome.sph.umich.edu/wiki/METAL;

16 Michigan Imputation Server, https://imputationserver.sph.umich.edu/;

17 FUMA, http://fuma.ctglab.nl/;

18 MGAMA, https://ctg.cncr.nl/software/magma;

19 H-MAGMA, https:/github.com/thewonlab/H-MAGMA;

20 LDSC, https://github.com/bulik/ldsc/;

21 LCV, https://github.com/lukejoconnor/LCV/;

22 DEPICT, https://github.com/perslab/depict;

23 FINDOR, https://github.com/gkichaev/FINDOR;

24 SuSiE, https://github.com/stephenslab/susieR;

25 PolyFun, https://github.com/omerwe/polyfun;

26 NHGRI-EBI GWAS Catalog, https://www.ebi.ac.uk/gwas/home;

27 The atlas of GWAS Summary Statistics, http://atlas.ctglab.nl/;

\section{METHODS}

30 Methods are available in the Methods section.

31 Note: One supplementary information pdf file, one supplementary figure pdf file, and

32 one supplementary table zip file are available. 


\section{ACKNOWLEDGEMENTS}

3 This research was partially supported by U.S. NIH grants MH086633 (H.Z.), MH116527

4 (TF.L.), and HD079124 (Y.L.). We thank Sophia Cui, Xiaopeng Zong, and Peter Vandehaar

5 for helpful conversations. We thank the individuals represented in the UK Biobank, $6 \mathrm{ABCD}, \mathrm{HCP}, \mathrm{PING}$, and PNC studies for their participation and the research teams for

7 their work in collecting, processing and disseminating these datasets for analysis. We

8 gratefully acknowledge all the studies and databases that made GWAS summary data

9 available. This research has been conducted using the UK Biobank resource (application 10 number 22783), subject to a data transfer agreement. Part of the data collection and

11 sharing for this project was funded by the Pediatric Imaging, Neurocognition and

12 Genetics Study (PING) (U.S. National Institutes of Health Grant RC2DA029475). PING is

13 funded by the National Institute on Drug Abuse and the Eunice Kennedy Shriver

14 National Institute of Child Health \& Human Development. PING data are disseminated 15 by the PING Coordinating Center at the Center for Human Development, University of 16 California, San Diego. Support for the collection of the PNC datasets was provided by

17 grant RC2MH089983 awarded to Raquel Gur and RC2MH089924 awarded to Hakon

18 Hakonarson. All PNC subjects were recruited through the Center for Applied Genomics

19 at The Children's Hospital in Philadelphia. Part of the data used in the preparation of this

20 article were obtained from the Adolescent Brain Cognitive Development (ABCD) Study

21 (https://abcdstudy.org), held in the NIMH Data Archive (NDA). This is a multisite,

22 longitudinal study designed to recruit more than 10,000 children age 9-10 and follow

23 them over 10 years into early adulthood. The ABCD Study is supported by the National

24 Institutes of Health and additional federal partners under award numbers

25 U01DA041022, U01DA041028, U01DA041048, U01DA041089, U01DA041106,

26 U01DA041117, U01DA041120, U01DA041134, U01DA041148, U01DA041156,

27 U01DA041174, U24DA041123, U24DA041147, U01DA041093, and U01DA041025. A full

28 list of supporters is available at https://abcdstudy.org/federal-partners.html. A listing of

29 participating sites and a complete listing of the study investigators can be found at 30 https://abcdstudy.org/scientists/workgroups/. ABCD consortium investigators designed

31 and implemented the study and/or provided data but did not necessarily participate in

32 analysis or writing of this report. This manuscript reflects the views of the authors and 
1 may not reflect the opinions or views of the NIH or ABCD consortium investigators. HCP

2 data were provided by the Human Connectome Project, WU-Minn Consortium (Principal

3 Investigators: David Van Essen and Kamil Ugurbil; 1U54MH091657) funded by the 16

4 NIH Institutes and Centers that support the NIH Blueprint for Neuroscience Research;

5 and by the McDonnell Center for Systems Neuroscience at Washington University.

6

7 AUTHOR CONTRIBUTIONS

8 B.Z., H.Z., Y.L., and J.L.S. designed the study. B.Z., TF. L, Y.Y., X.W., and TY. L analyzed the

9 data. TF. L, Y.S., Z.Z., Y.Y., X.W., TY. L, and D.X., downloaded the datasets, preprocessed

$10 \mathrm{dMRI}$ data, and undertook the quantity controls. P.R., M.E.H., J.B., and J.F.F. analyzed

11 brain cell chromatin accessibility data. B.Z. and H.Z. wrote the manuscript with feedback

12 from all authors.

13

14 CORRESPINDENCE AND REQUESTS FOR MATERIALS should be addressed to H.Z.

16 COMPETETING FINANCIAL INTERESTS

17 The authors declare no competing financial interests.

\section{REFERENCES}

21 1. van den Heuvel, M.P. \& Sporns, O. A cross-disorder connectome landscape of

22 brain dysconnectivity. Nature reviews neuroscience 20, 435-446 (2019).

232 2. Hagmann, P. et al. Mapping the structural core of human cerebral cortex. PLoS

24 biology 6(2008).

25 3. Zielinski, B.A., Gennatas, E.D., Zhou, J. \& Seeley, W.W. Network-level structural covariance in the developing brain. Proceedings of the National Academy of Sciences 107, 18191-18196 (2010).

28 4. Fields, R.D. White matter in learning, cognition and psychiatric disorders. Trends 29 in neurosciences 31, 361-370 (2008).

30 5. Filley, C.M. \& Fields, R.D. White matter and cognition: making the connection. Journal of neurophysiology 116, 2093-2104 (2016). 
1 6. Kuceyeski, A., Maruta, J., Relkin, N. \& Raj, A. The Network Modification (NeMo) Tool: elucidating the effect of white matter integrity changes on cortical and subcortical structural connectivity. Brain connectivity 3, 451-463 (2013).

7. Bathelt, J., Scerif, G., Nobre, A. \& Astle, D. Whole-brain white matter organization, intelligence, and educational attainment. Trends in neuroscience and education 15, 38-47 (2019).

8. Vaquero, L., Rodríguez-Fornells, A. \& Reiterer, S.M. The left, the better: white-matter brain integrity predicts foreign language imitation ability. Cerebral Cortex 27, 3906-3917 (2017).

9. Wu, Z.-M. et al. White matter microstructural alterations in children with ADHD: categorical and dimensional perspectives. Neuropsychopharmacology 42, $572-580$ (2017).

10. Zou, K. et al. Alterations of white matter integrity in adults with major depressive disorder: a magnetic resonance imaging study. Journal of psychiatry \& neuroscience: JPN 33, 525 (2008).

11. Cetin-Karayumak, S. et al. White matter abnormalities across the lifespan of schizophrenia: a harmonized multi-site diffusion MRI study. Molecular psychiatry, 1-12 (2019).

12. Versace, A. et al. Elevated left and reduced right orbitomedial prefrontal fractional anisotropy in adults with bipolar disorder revealed by tract-based spatial statistics. Archives of general psychiatry 65, 1041-1052 (2008).

13. De Santis, S. et al. Evidence of early microstructural white matter abnormalities in multiple sclerosis from multi-shell diffusion MRI. Neurolmage: Clinical 22, 101699 (2019).

14. Lee, S. et al. White matter hyperintensities are a core feature of Alzheimer's disease: evidence from the dominantly inherited Alzheimer network. Annals of neurology 79, 929-939 (2016).

15. Hess, C., Christine, C., Apple, A., Dillon, W. \& Aminoff, M. Changes in the thalamus in atypical parkinsonism detected using shape analysis and diffusion tensor imaging. American Journal of Neuroradiology 35, 897-903 (2014). 
1 16. Veselý, B. \& Rektor, I. The contribution of white matter lesions (WML) to Parkinson's disease cognitive impairment symptoms: a critical review of the literature. Parkinsonism \& related disorders 22, S166-S170 (2016).

17. Basser, P.J., Mattiello, J. \& LeBihan, D. Estimation of the effective self-diffusion tensor from the NMR spin echo. Journal of Magnetic Resonance, Series B 103, 247-254 (1994).

18. Grieve, S., Williams, L., Paul, R., Clark, C. \& Gordon, E. Cognitive aging, executive function, and fractional anisotropy: a diffusion tensor MR imaging study. American Journal of Neuroradiology 28, 226-235 (2007).

19. Basser, P. \& Pierpaoli, C. Microstructural and physiological features of tissues elucidated by quantitative-diffusion-tensor MRI. Journal of magnetic resonance. Series B 111, 209 (1996).

20. Ennis, D.B. \& Kindlmann, G. Orthogonal tensor invariants and the analysis of diffusion tensor magnetic resonance images. Magnetic Resonance in Medicine: An Official Journal of the International Society for Magnetic Resonance in Medicine 55, 136-146 (2006).

21. Mascalchi, M. et al. Progression of microstructural damage in spinocerebellar ataxia type 2: a longitudinal DTI study. American Journal of Neuroradiology 36, 1096-1101 (2015).

22. Vuoksimaa, E. et al. Heritability of white matter microstructure in late middle age: A twin study of tract-based fractional anisotropy and absolute diffusivity indices. Human brain mapping 38, 2026-2036 (2017).

23. Elliott, L.T. et al. Genome-wide association studies of brain imaging phenotypes in UK Biobank. Nature 562, 210-216 (2018).

24. Kochunov, P. et al. Heritability of fractional anisotropy in human white matter: a comparison of Human Connectome Project and ENIGMA-DTI data. Neuroimage 111, 300-311 (2015).

25. Zhao, B. et al. Large-scale GWAS reveals genetic architecture of brain white matter microstructure and genetic overlap with cognitive and mental health traits $(n=17,706)$. Molecular Psychiatry (2019). 
1 26. Rutten-Jacobs, L.C. et al. Genetic study of white matter integrity in UK Biobank $(\mathrm{N}=8448)$ and the overlap with stroke, depression, and dementia. Stroke 49, 1340-1347 (2018).

4 27. Traylor, M. et al. Genetic variation in PLEKHG1 is associated with white matter hyperintensities ( $n=11,226)$. Neurology 92, e749-e757 (2019).

28. Fornage, M. et al. Genome-wide association studies of cerebral white matter lesion burden: the CHARGE consortium. Annals of neurology 69, 928-939 (2011).

29. Adib-Samii, P. et al. 17q25 Locus is associated with white matter hyperintensity volume in ischemic stroke, but not with lacunar stroke status. Stroke 44,

11 30. O'Connor, L.J. et al. Extreme polygenicity of complex traits is explained by negative selection. The American Journal of Human Genetics 105, 456-476 (2019).

31. Adib-Samii, P. et al. Genetic architecture of white matter hyperintensities differs in hypertensive and nonhypertensive ischemic stroke. Stroke 46, 348-353 (2015).

32. Safadi, Z. et al. Functional segmentation of the anterior limb of the internal capsule: linking white matter abnormalities to specific connections. Journal of Neuroscience 38, 2106-2117 (2018).

33. Sudlow, C. et al. UK biobank: an open access resource for identifying the causes of a wide range of complex diseases of middle and old age. PLoS Medicine 12,

34. Casey, B. et al. The adolescent brain cognitive development ( $A B C D)$ study: imaging acquisition across 21 sites. Developmental cognitive neuroscience 32, 43-54 (2018).

25 35. Somerville, L.H. et al. The Lifespan Human Connectome Project in Development: A large-scale study of brain connectivity development in 5-21 year olds. Neurolmage 183, 456-468 (2018).

28 36. Jernigan, T.L. et al. The pediatric imaging, neurocognition, and genetics (PING) data repository. Neuroimage 124, 1149-1154 (2016).

30 37. Satterthwaite, T.D. et al. Neuroimaging of the Philadelphia neurodevelopmental cohort. Neuroimage 86, 544-553 (2014). 
1 38. Jahanshad, N. et al. Multi-site genetic analysis of diffusion images and voxelwise heritability analysis: A pilot project of the ENIGMA-DTI working group. Neuroimage 81, 455-469 (2013).

4 39. Kochunov, P. et al. Multi-site study of additive genetic effects on fractional anisotropy of cerebral white matter: comparing meta and megaanalytical approaches for data pooling. Neuroimage 95, 136-150 (2014).

40. Hall, P., Müller, H.-G. \& Wang, J.-L. Properties of principal component methods for functional and longitudinal data analysis. The annals of statistics, 1493-1517 (2006).

41. Mattingsdal, M. et al. Pathway analysis of genetic markers associated with a functional MRI faces paradigm implicates polymorphisms in calcium responsive pathways. Neuroimage 70, 143-149 (2013).

42. Azadeh, S. et al. Integrative Bayesian analysis of neuroimaging-genetic data through hierarchical dimension reduction. in 2016 IEEE 13th International Symposium on Biomedical Imaging (ISBI) 824-828 (IEEE, 2016).

43. Yang, J., Lee, S.H., Goddard, M.E. \& Visscher, P.M. GCTA: a tool for genome-wide complex trait analysis. The American Journal of Human Genetics $\mathbf{8 8}, 76-82$ (2011).

44. Watanabe, K., Taskesen, E., Bochoven, A. \& Posthuma, D. Functional mapping and annotation of genetic associations with FUMA. Nature Communications $\mathbf{8}$, 1826 (2017).

45. Wang, G., Sarkar, A.K., Carbonetto, P. \& Stephens, M. A simple new approach to variable selection in regression, with application to genetic fine-mapping. bioRxiv, 501114 (2019).

25 46. Weissbrod, O. et al. Functionally-informed fine-mapping and polygenic localization of complex trait heritability. BioRxiv, 807792 (2019).

27 47. Martin, A.R., Daly, M.J., Robinson, E.B., Hyman, S.E. \& Neale, B.M. Predicting polygenic risk of psychiatric disorders. Biological psychiatry 86, 97-109 (2019).

29 48. Bulik-Sullivan, B. et al. An atlas of genetic correlations across human diseases and traits. Nature Genetics 47, 1236-1241 (2015). 
49. Jansen, P.R. et al. Genome-wide analysis of insomnia in 1,331,010 individuals identifies new risk loci and functional pathways. Nature Genetics 51, 394-403 (2019).

50. Skol, A.D., Scott, L.J., Abecasis, G.R. \& Boehnke, M. Joint analysis is more efficient than replication-based analysis for two-stage genome-wide association studies. Nature Genetics 38, 209-213 (2006).

51. Jansen, I.E. et al. Genome-wide meta-analysis identifies new loci and functional pathways influencing Alzheimer's disease risk. Nature genetics 51, 404-413 (2019).

52. Bycroft, C. et al. The UK Biobank resource with deep phenotyping and genomic data. Nature 562, 203-209 (2018).

53. Kichaev, G. et al. Leveraging polygenic functional enrichment to improve GWAS power. The American Journal of Human Genetics 104, 65-75 (2019).

54. Buniello, A. et al. The NHGRI-EBI GWAS Catalog of published genome-wide association studies, targeted arrays and summary statistics 2019. Nucleic Acids Research 47, D1005-D1012 (2018).

55. van der Meer, D. et al. Brain scans from 21,297 individuals reveal the genetic architecture of hippocampal subfield volumes. Molecular Psychiatry, in press. (2018).

56. Verhaaren, B.F. et al. Multiethnic genome-wide association study of cerebral white matter hyperintensities on MRI. Circulation: Cardiovascular Genetics 8, 398-409 (2015).

57. Vojinovic, D. et al. Genome-wide association study of 23,500 individuals identifies 7 loci associated with brain ventricular volume. Nature communications 9, 1-11 (2018).

58. Klein, M. et al. Genetic markers of ADHD-related variations in intracranial volume. American Journal of Psychiatry 176, 228-238 (2019).

59. Hibar, D.P. et al. Common genetic variants influence human subcortical brain structures. Nature 520, 224-229 (2015).

60. Luo, Q. et al. Association of a Schizophrenia-Risk Nonsynonymous Variant With Putamen Volume in Adolescents: A Voxelwise and Genome-Wide Association Study. JAMA psychiatry 76, 435-445 (2019). 
1 61. Hashimoto, R. et al. Common variants at $1 \mathrm{p} 36$ are associated with superior frontal gyrus volume. Translational psychiatry 4, e472-e472 (2014).

3 62. Ikram, M.A. et al. Common variants at $6 q 22$ and $17 q 21$ are associated with intracranial volume. Nature Genetics 44, 539-544 (2012).

63. Sprooten, E. et al. Common genetic variants and gene expression associated with white matter microstructure in the human brain. Neuroimage 97, 252-261 (2014).

64. Shen, L. et al. Whole genome association study of brain-wide imaging phenotypes for identifying quantitative trait loci in $\mathrm{MCl}$ and $\mathrm{AD}$ : A study of the ADNI cohort. Neuroimage 53, 1051-1063 (2010).

11 65. Chung, J. et al. Genome-wide association study of Alzheimer's disease endophenotypes at prediagnosis stages. Alzheimer's \& Dementia 14, 623-633 (2018).

66. Chen, C.-H. et al. Leveraging genome characteristics to improve gene discovery for putamen subcortical brain structure. Scientific Reports 7, 15736 (2017).

16 67. Christopher, L. et al. A variant in PPP4R3A protects against alzheimer-related metabolic decline. Annals of neurology 82, 900-911 (2017).

68. Luciano, M. et al. Association analysis in over 329,000 individuals identifies 116 independent variants influencing neuroticism. Nature Genetics 50, 6-11 (2018).

69. Baselmans, B.M. et al. Multivariate genome-wide analyses of the well-being spectrum. Nature genetics 51, 445-451 (2019).

70. Linnér, R.K. et al. Genome-wide association analyses of risk tolerance and risky behaviors in over 1 million individuals identify hundreds of loci and shared genetic influences. Nature Genetics 51, 245-257 (2019).

71. Davies, G. et al. Study of 300,486 individuals identifies 148 independent genetic

28 72. Lee, J.J. et al. Gene discovery and polygenic prediction from a genome-wide association study of educational attainment in 1.1 million individuals. Nature Genetics 50, 1112-1121 (2018). 
1 73. Ikeda, M. et al. Genome-wide association study detected novel susceptibility

2 genes for schizophrenia and shared trans-populations/diseases genetic effect.

3 Schizophrenia bulletin 45, 824-834 (2019).

4 74. Hyde, C.L. et al. Identification of 15 genetic loci associated with risk of major

5 depression in individuals of European descent. Nature genetics 48, 1031 (2016).

6 75. Ruderfer, D.M. et al. Polygenic dissection of diagnosis and clinical dimensions of bipolar disorder and schizophrenia. Molecular psychiatry 19, 1017 (2014).

8 76. Lesch, K.-P. et al. Molecular genetics of adult ADHD: converging evidence from $9 \quad$ genome-wide association and extended pedigree linkage studies. Journal of neural transmission 115, 1573-1585 (2008).

11 77. Grove, J. et al. Identification of common genetic risk variants for autism spectrum disorder. Nature genetics 51, 431 (2019).

13 78. Allen, H.L. et al. Hundreds of variants clustered in genomic loci and biological pathways affect human height. Nature 467, 832-838 (2010).

15 79. Morris, J.A. et al. An atlas of genetic influences on osteoporosis in humans and mice. Nature genetics 51, 258-266 (2019).

80. Medina-Gomez, C. et al. Life-course genome-wide association study meta-analysis of total body BMD and assessment of age-specific effects. The American Journal of Human Genetics 102, 88-102 (2018).

81. Liu, M. et al. Association studies of up to 1.2 million individuals yield new insights into the genetic etiology of tobacco and alcohol use. Nature genetics 51, 237-244 (2019).

82. Sanchez-Roige, S. et al. Genome-wide association study meta-analysis of the Alcohol Use Disorders Identification Test (AUDIT) in two population-based cohorts. American Journal of Psychiatry, appi. ajp. 2018.18040369 (2018).

83. Kouri, N. et al. Genome-wide association study of corticobasal degeneration identifies risk variants shared with progressive supranuclear palsy. Nature communications 6, 7247 (2015).

29 84. Simon-Sanchez, J. et al. Genome-wide association study reveals genetic risk underlying Parkinson's disease. Nature genetics 41, 1308 (2009). 
1 85. Kunkle, B.W. et al. Genetic meta-analysis of diagnosed Alzheimer's disease identifies new risk loci and implicates $A \beta$, tau, immunity and lipid processing. Nature genetics 51, 414 (2019).

4 86. Beecham, A.H. et al. Analysis of immune-related loci identifies 48 new susceptibility variants for multiple sclerosis. Nature genetics 45, 1353 (2013).

87. Dashti, H. et al. GWAS in 446,118 European adults identifies 78 genetic loci for self-reported habitual sleep duration supported by accelerometer-derived estimates. bioRxiv, 274977 (2018).

9 88. Jones, S.E. et al. Genome-wide association analyses of chronotype in 697,828 individuals provides insights into circadian rhythms. Nature communications 10 , 343 (2019).

89. Melin, B.S. et al. Genome-wide association study of glioma subtypes identifies specific differences in genetic susceptibility to glioblastoma and non-glioblastoma tumors. Nature genetics 49, 789 (2017).

90. Kinnersley, B. et al. Genome-wide association study identifies multiple

91. Traylor, M. et al. Genetic risk factors for ischaemic stroke and its subtypes (the METASTROKE collaboration): a meta-analysis of genome-wide association studies. The Lancet Neurology 11, 951-962 (2012).

92. Malik, R. et al. Multiancestry genome-wide association study of 520,000 subjects identifies 32 loci associated with stroke and stroke subtypes. Nature genetics $\mathbf{5 0}$, 524-537 (2018).

93. Dichgans, M. et al. Shared genetic susceptibility to ischemic stroke and coronary artery disease: a genome-wide analysis of common variants. Stroke 45, 24-36 (2014)

26 94. Anney, R.J.L. et al. Meta-analysis of GWAS of over 16,000 individuals with autism spectrum disorder highlights a novel locus at 10q24.32 and a significant overlap with schizophrenia. Molecular Autism 8, 21 (2017).

29 95. Chang, D. et al. A meta-analysis of genome-wide association studies identifies 17 new Parkinson's disease risk loci. Nature genetics 49, 1511 (2017). 
1 96. Nagel, M. et al. Meta-analysis of genome-wide association studies for neuroticism in 449,484 individuals identifies novel genetic loci and pathways. Nature Genetics 50, 920 (2018).

4 97. Lam, M. et al. Large-Scale Cognitive GWAS Meta-Analysis Reveals Tissue-Specific Neural Expression and Potential Nootropic Drug Targets. Cell reports 21, 2597-2613 (2017).

98. Rask-Andersen, M., Karlsson, T., Ek, W.E. \& Johansson, Å. Genome-wide

99. Onnink, A.M.H. et al. Deviant white matter structure in adults with attention-deficit/hyperactivity disorder points to aberrant myelination and affects neuropsychological performance. Progress in Neuro-Psychopharmacology and Biological Psychiatry 63, 14-22 (2015).

100. Wen, Q. et al. White matter alterations in early-stage Alzheimer's disease: A

101. Seok, J.-H. et al. White matter abnormalities associated with auditory

102. Minn, Y., Suk, S. \& Do, S. Osteoporosis as an independent risk factor for silent brain infarction and white matter changes in men and women: the PRESENT project. Osteoporosis International 25, 2465-2469 (2014).

103. Hannawi, Y. et al. Hypertension is associated with white matter disruption in apparently healthy middle-aged individuals. American Journal of Neuroradiology

105. Dodd, J.W. et al. Brain structure and function in chronic obstructive pulmonary 39, 2243-2248 (2018). disease: a multimodal cranial magnetic resonance imaging study. American journal of respiratory and critical care medicine 186, 240-245 (2012). 
1 106. Berry, C. et al. Small-vessel disease in the heart and brain: current knowledge, unmet therapeutic need, and future directions. Journal of the American Heart Association 8, e011104 (2019).

$4 \quad$ 107. Xu, J., Li, Y., Lin, H., Sinha, R. \& Potenza, M.N. Body mass index correlates negatively with white matter integrity in the fornix and corpus callosum: a diffusion tensor imaging study. Human brain mapping 34, 1044-1052 (2013).

7 108. O'Connor, L.J. \& Price, A.L. Distinguishing genetic correlation from causation across 52 diseases and complex traits. Nature genetics 50, 1728-1734 (2018).

9 109. Cortes, A., Albers, P.K., Dendrou, C.A., Fugger, L. \& McVean, G. Identifying cross-disease components of genetic risk across hospital data in the UK Biobank. Nature Genetics 52, 126-134 (2020).

12 110. de Leeuw, C.A., Mooij, J.M., Heskes, T. \& Posthuma, D. MAGMA: generalized gene-set analysis of GWAS data. PLoS Computational Biology 11, e1004219 (2015).

15 111. Hofer, E. et al. White matter lesion progression: genome-wide search for genetic influences. Stroke 46, 3048-3057 (2015).

112. Sprooten, E. et al. White matter integrity as an intermediate phenotype: exploratory genome-wide association analysis in individuals at high risk of bipolar disorder. Psychiatry Research 206, 223-231 (2013).

113. Hoffmann, T.J. et al. Genome-wide association analyses using electronic health records identify new loci influencing blood pressure variation. Nature genetics 49, 54 (2017).

114. Hill, W.D. et al. Genetic contributions to two special factors of neuroticism are associated with affluence, higher intelligence, better health, and longer life. Molecular psychiatry, 1-19 (2019).

115. Sey, N.Y. et al. A computational tool (H-MAGMA) for improved prediction of brain-disorder risk genes by incorporating brain chromatin interaction profiles. (Nature Publishing Group, 2020). schizophrenia across 4322 individuals: results from the ENIGMA Schizophrenia DTI Working Group. Molecular psychiatry 23, 1261 (2018). 
$1 \quad$ 117. Stämpfli, P. et al. Subtle white matter alterations in schizophrenia identified with

2 a new measure of fiber density. Scientific reports 9, 1-11 (2019).

3 118. Hamza, T.H. et al. Common genetic variation in the HLA region is associated with

$4 \quad$ late-onset sporadic Parkinson's disease. Nature genetics 42, 781 (2010).

5 119. Hol, E. et al. Neuronal expression of GFAP in patients with Alzheimer pathology and identification of novel GFAP splice forms. Molecular psychiatry 8, 786-796 (2003).

8 120. Jakkula, E. et al. Genome-wide association study in a high-risk isolate for multiple sclerosis reveals associated variants in STAT3 gene. The American Journal of Human Genetics 86, 285-291 (2010).

11 121. Finucane, H.K. et al. Partitioning heritability by functional annotation using genome-wide association summary statistics. Nature genetics 47, 1228-1235 (2015).

122. Kundaje, A. et al. Integrative analysis of 111 reference human epigenomes. Nature 518, 317 (2015).

16 123. Fullard, J.F. et al. An atlas of chromatin accessibility in the adult human brain. Genome research 28, 1243-1252 (2018).

18 124. Aguet, F. et al. The GTEx Consortium atlas of genetic regulatory effects across human tissues. BioRxiv, 787903 (2019).

20 125. Wang, Q. et al. A Bayesian framework that integrates multi-omics data and gene networks predicts risk genes from schizophrenia GWAS data. Nature neuroscience 22, 691 (2019).

23 126. Uribe-Bojanini, E., Hernandez-Quiceno, S. \& Cock-Rada, A.M. Xeroderma Pigmentosum with Severe Neurological Manifestations/De Sanctis-Cacchione

127. Kraemer, K.H. et al. Xeroderma pigmentosum, trichothiodystrophy and Cockayne syndrome: a complex genotype-phenotype relationship. Neuroscience 145, 1388-1396 (2007).

29 128. Anttinen, A. et al. Neurological symptoms and natural course of xeroderma pigmentosum. Brain 131, 1979-1989 (2008). 
1 129. Kassubek, J. et al. The cerebro-morphological fingerprint of a progeroid

140. syndrome: white matter changes correlate with neurological symptoms in xeroderma pigmentosum. PloS one 7(2012).

130. Harreld, J., Smith, E., Prose, N., Puri, P. \& Barboriak, D. Trichothiodystrophy with dysmyelination and central osteosclerosis. American journal of neuroradiology 31, 129-130 (2010).

131. Menning, S. et al. Changes in brain white matter integrity after systemic treatment for breast cancer: a prospective longitudinal study. Brain imaging and behavior 12, 324-334 (2018).

132. Deprez, S., Billiet, T., Sunaert, S. \& Leemans, A. Diffusion tensor MRI of chemotherapy-induced cognitive impairment in non-CNS cancer patients: a review. Brain imaging and behavior 7, 409-435 (2013).

133. Hamanaka, G., Ohtomo, R., Takase, H., Lok, J. \& Arai, K. White-matter repair: Interaction between oligodendrocytes and the neurovascular unit. Brain circulation 4, 118 (2018).

134. Blalock, E.M. et al. Incipient Alzheimer's disease: microarray correlation analyses reveal major transcriptional and tumor suppressor responses. Proceedings of the National Academy of Sciences 101, 2173-2178 (2004).

135. Koini, M., Rombouts, S., Veer, I., Van Buchem, M. \& Huijbregts, S. White matter microstructure of patients with neurofibromatosis type 1 and its relation to inhibitory control. Brain imaging and behavior 11, 1731-1740 (2017).

136. Kang, M. \& Lee, Y.-S. The impact of RASopathy-associated mutations on CNS development in mice and humans. Molecular brain 12, 1-17 (2019).

137. Shikanai, M., Yuzaki, M. \& Kawauchi, T. Rab family small GTPases-mediated regulation of intracellular logistics in neural development. Histology and Histopathology 33, 765-771 (2017).

138. Qu, L. et al. The Ras Superfamily of Small GTPases in Non-neoplastic Cerebral Diseases. Frontiers in Molecular Neuroscience 12, 121 (2019).

139. Govek, E.-E., Newey, S.E. \& Van Aelst, L. The role of the Rho GTPases in neuronal development. Genes \& development 19, 1-49 (2005).

\section{Pers, T.H. et al. Biological interpretation of genome-wide association studies} using predicted gene functions. Nature Communications 6, 5890 (2015). 
1 141. Smith, S.M. \& Nichols, T.E. Statistical challenges in "big data" human neuroimaging. Neuron 97, 263-268 (2018).

3 142. Martin, A.R. et al. Clinical use of current polygenic risk scores may exacerbate $4 \quad$ health disparities. Nature genetics 51, 584 (2019).

5 143. Duncan, L. et al. Analysis of polygenic risk score usage and performance in diverse human populations. Nature communications 10, 1-9 (2019).

7 144. Xu, Q. et al. CHIMGEN: a Chinese imaging genetics cohort to enhance cross-ethnic and cross-geographic brain research. Molecular Psychiatry, 1-13

9 (2019).

$10145 . \quad$ Chen, C.-Y. et al. Improved ancestry inference using weights from external reference panels. Bioinformatics 29, 1399-1406 (2013).

12 146. Jiang, L. et al. A resource-efficient tool for mixed model association analysis of 13 large-scale data. Nature genetics 51, 1749 (2019).

14 147. Purcell, S. et al. PLINK: a tool set for whole-genome association and population-based linkage analyses. The American Journal of Human Genetics 81, 559-575 (2007).

17 148. Willer, C.J., Li, Y. \& Abecasis, G.R. METAL: fast and efficient meta-analysis of genomewide association scans. Bioinformatics 26, 2190-2191 (2010).

149. Consortium, I.H. Integrating common and rare genetic variation in diverse human populations. Nature 467, 52-58 (2010).

21 150. Wang, D. et al. Comprehensive functional genomic resource and integrative model for the human brain. Science 362, eaat8464 (2018).

23 151. Won, H. et al. Chromosome conformation elucidates regulatory relationships in developing human brain. Nature 538, 523-527 (2016).

25 152. Liberzon, A. et al. Molecular signatures database (MSigDB) 3.0. Bioinformatics 27, $1739-1740$ (2011).

30 GWAS design and Imaging phenotypes. We analyzed the following GWAS datasets

31 separately: 1) the UKB British discovery GWAS, which used data of individuals of British 32 ancestry ${ }^{52}$ from the UKB study ( $\left.n=33,292\right)$; 2 ) five validation GWAS performed on 
1 individuals of European ancestry: UKB White but Non-British (UKBW, $n=1,809$ ), ABCD

2 European (ABCDE, $n=3,821)$, HCP $(n=334)$, PING $(n=461)$, and PNC $(n=537) ; 3)$ two

3 non-European UKB validation GWAS: UKB Asian (UKBA, $n=419$ ) and UKB Black (UKBBL,

$4 \quad n=211)$; 4) two non-European non-UKB validation GWAS, including ABCD Hispanic

$5(\mathrm{ABCDH}, n=768)$ and $\mathrm{ABCD}$ African American (ABCDA, $n=1,257)$; and 5) a UKB British

6 GWAS with subjects not present in previous GWAS $^{25}$ (also removed the relatives of

7 previous GWAS subjects, $n=15,214)$. See Supplementary Table $\mathbf{4 0}$ for a summary of

8 these GWAS and demographic information of study cohorts. The raw dMRI, covariates

9 and genetic data were downloaded from each data resource. We processed the dMRI

10 data locally using consistent procedures via ENIGMA-DTI pipeline ${ }^{38,39}$ to generate 215

11 mean and PC DTI phenotypes for 21 predefined white matter tracts (Supplementary

12 Table 41). A full description of image acquisition and preprocessing, quality controls,

13 ENIGMA-DTI pipeline, white matter tracts, principle component extraction, and

14 formulas of DTI parameters are detailed in Supplementary Note. An overview of tract

15 annotation and imaging procedures is shown in Supplementary Figures 24-26 and a few

16 image examples are given in Supplementary Figures 27-30. For each continuous

17 phenotype or covariate variable, we removed values greater than five times the median

18 absolute deviation from the median value. The ancestry assignment in UKB was based

19 on self-reported ethnic background (Data-Field 21000), whose accuracy was verified in

20 Bycroft, et al. ${ }^{52}$ For $A B C D$, we assigned ancestry by a combination analysis using

21 self-reported ethnicity and ancestry inference results from SNPweights ${ }^{145}$, see

22 Supplementary Note for details.

Association discovery and validation. Genotyping and quality controls are documented

25 in Supplementary Note. We estimated the SNP heritability by all autosomal SNPs in UKB

26 British discovery GWAS data using GCTA-GREML analysis ${ }^{43}$. The adjusted covariates

27 included age (at imaging), age-squared, sex, age-sex interaction, age-squared-sex

28 interaction, imaging site, as well as the top 40 genetic principle components (PCs)

29 provided by $U_{K B}^{52}$ (Data-Field 22009). The heritability estimates were tested in

30 one-sided likelihood ratio tests. We performed linear mixed model-based association

31 analysis using fastGWA ${ }^{146}$. The same set of covariates as in GCTA-GREML analysis were

32 adjusted. To replicate previous findings, we also performed another UKB British GWAS 
1 with subjects not present in previous GWAS $^{25}$. In addition, GWAS were separately

2 performed on European validation datasets UKBW, ABCDE, HCP, PING, and PNC using

3 Plink $^{147}$. In the five validation GWAS, we adjusted for age, age-squared, sex, age-sex

4 interaction, age-squared-sex interaction, and top ten genetic PCs estimated from

5 genetic variants. We also adjusted for imaging sites in $A B C D$ analysis. The meta-analysis

6 was then performed on these validation datasets using METAL ${ }^{148}$ with the sample-size

7 weighted approach.

9 We applied a few analyses to support the findings in UKB British discovery GWAS. First, 10 the $\operatorname{LDSC}^{48}$ software (version 1.0.0) was used to estimate the pairwise genetic 11 correlation between DTI parameter values in discovery GWAS and the meta-analyzed 12 five European validation GWAS ( $n=6,962)$. We used the pre-calculated LD scores 13 provided by LDSC, which were computed using 1000 Genomes European data. We used 14 HapMap3 $^{149}$ variants and removed all variants in the major histocompatibility complex 15 (MHC) region. In addition, we performed another meta-analysis for the UKB British 16 discovery GWAS and the five European validation GWAS to check whether the $P$-values

17 became smaller after combining these results. Next, polygenic risk scores (PRS) were 18 created on nine validation datasets using the BLUP effect sizes estimated from 19 GCTA-GREML analysis of UKB British discovery GWAS. We used PLINK to generate risk 20 scores in each testing data by summarizing across genome-wide variants, weighed by 21 their BLUP effect sizes. We tried $17 P$-value thresholds for variant selection using their 22 marginal $P$-values from fastGWA: 1, 0.8, 0.5, 0.4, 0.3, 0.2, 0.1, 0.08, 0.05, 0.02, 0.01, $1 \times$ $2310^{-3}, 1 \times 10^{-4}, 1 \times 10^{-5}, 1 \times 10^{-6}, 1 \times 10^{-7}$, and $1 \times 10^{-8}$. Then, we generated 17 polygenic 24 profiles for each phenotype and reported the best prediction power that can be 25 achieved by a single profile. The association between polygenic profile and phenotype 26 was estimated and tested in linear models, adjusting for the effects of age, gender, and

27 top ten genetic PCs. The additional phenotypic variation that can be explained by 28 polygenic profile (i.e., the incremental R-squared) was used to measure the prediction 29 accuracy.

31 Genomic risk loci characterization and comparison with previous findings. We defined 32 genomic risk loci by using FUMA (version 1.3.5e). We input the UKB British discovery 
1 GWAS summary statistics after reweighting the $P$-values using functional information via

2 FINDOR $^{53}$. Specifically, FUMA first clumped partially independent significant variants,

3 which were variants with a $P$-value smaller than the predefined threshold and

4 independent of other significant variants (LD $r^{2}<0.6$, default value). FUMA constructed

5 LD blocks for these independent significant variants by tagging all variants in $\operatorname{LD}\left(r^{2} \geq\right.$

6 0.6) with at least one independent significant variant and had a MAF $\geq 0.0005$. These

7 variants included those from the 1000 Genomes reference panel that may not have

8 been included in the GWAS. Based on these significant variants, independent lead

9 variants were identified as those that were independent from each other ( $\left.L D r^{2}<0.1\right)$. If

10 LD blocks of independent significant variants were closed ( $<250 \mathrm{~kb}$ based on the closest

11 boundary variants of LD blocks), they were merged to a single genomic locus. Thus, each

12 genomic risk locus could contain more than one independent significant variants and

13 lead variants. We performed functionally-informed fine-mapping by using SuSiE ${ }^{45}$

14 method via PolyFun ${ }^{46}$ framework for risk loci. The summary statistics from UKB British

15 discovery GWAS were used as input. As suggested, we estimated the LD matrix using

16 our training GWAS individuals. To validate previous findings reported in Zhao, et al. ${ }^{25}$,

17 we estimated the pairwise genetic correlation between DTI parameter values in

18 previous GWAS and the UKB British GWAS with subjects not included in previous GWAS.

19 We also estimated the replication slope ${ }^{53}$ between two groups of standardized effect

20 sizes. We focused on previously reported top $\left(P<1 \times 10^{-6}\right)$ independent SNPs after

21 LD-based clumping (window size 250, LD $r^{2}=0.01$ ). Independent significant variants and

22 all their tagged variants were searched by FUMA in the NHGRI-EBI GWAS catalog

23 (version 2019-09-24) to look for previously reported associations $\left(P<9 \times 10^{-6}\right)$ with any

24 traits. In our UKB British discovery GWAS data, we performed voxel-wise association

25 analysis to illustrate spatial maps for several selected pleiotropic variants. The same set

26 of covariates used in the above tract-based GWAS analysis were adjusted in this

27 voxel-wise analysis.

29 Genetic correlation estimation and validation. We used LDSC to estimate the pairwise 30 genetic correlation between DTI parameters and other complex traits. The summary

31 statistics of DTI parameters were from the UKB British discovery GWAS and the

32 summary statistics of other traits were collected from publicly accessible data resources 
1 listed in Supplementary Table 18. To replicate the significant associations, we reran

2 LDSC using the meta-analyzed summary statistics from the five European validation

3 GWAS. In addition, we also constructed PRS for other complex traits on each of the five

4 validation datasets and tested whether the PRS had significant association with DTI

5 parameters. We used the LD-based pruning (window size 50 , step 5 , LD $r^{2}=0.2$ )

6 procedure to account for the LD structure in this cross-trait PRS analysis. We also

7 applied the 17 GWAS $P$-value thresholds for variants selection and reported the smallest

$8 \quad P$-value observed in validation data. We applied the LCV $^{108}$ (version 2019-03-14) to

9 explore the genetical causal relationships between DTI parameters and other complex

10 traits. We used meta-analyzed GWAS summary statistics and the pre-calculated LD

11 scores provided by LDSC.

13 Gene-level analysis. We first performed gene-based association analysis in UKB British 14 discovery GWAS for 18,796 protein-coding genes using MAGMA $^{110}$ (version 1.07). 15 Default MAGMA settings were used with zero window size around each gene. We then 16 carried out FUMA functional annotation and mapping analysis, in which variants were 17 annotated with their biological functionality and then were linked to 35,808 candidate 18 genes by a combination of positional, eQTL, and 3D chromatin interaction mappings. We 19 chose brain-related tissues/cells in all options and used default values for all other 20 parameters. For the detected genes in MAGMA and FUMA, we performed lookups in 21 the NHGRI-EBI GWAS catalog (version 2020-02-08) again to explore their previously 22 reported associations. We also applied H-MAGMA ${ }^{115}$ (version 2019-11-29) to perform $23 \mathrm{Hi}-\mathrm{C}$ coupled gene-based association analysis by integrating $\mathrm{Hi}-\mathrm{C}$ profiles from fetal and 24 adult brain tissues ${ }^{150,151}$.

26 Biological annotations. We performed heritability enrichment analysis via partitioned 27 LDSC $^{121}$. Baseline models were included when estimating the enrichment scores for our 28 tissue type and cell type specific annotations. Methods to prepare in-house chromatin 29 data of three glial cell subtypes and two neuronal cell subtypes can be found in the 30 Supplementary Note. We performed gene property analysis for the 13 GTEx ${ }^{124}$ v8 brain 31 tissues via MAGMA. Specifically, we tested whether the tissue-specific gene expression 32 levels can be linked to the strength of the gene-trait association. In addition, we treated 
1 DTI associated genes in MAGMA, H-MAGMA or FUMA analysis as an annotation and

2 tested whether the heritability of other complex traits was enriched in this DTI

3 annotation. MAGMA and DEPICT (version 1 rel194) were separately used to explore the

4 implicated biological pathways. MAGMA gene-set analysis examined 5,500 curated gene

5 sets and 9,996 Gene Ontology (GO) terms from the Molecular Signatures Database ${ }^{152}$

6 (MSigDB, version 7.0) and DEPICT tested 10,968 pre-constructed gene sets using GWAS

7 summary statistics with $P$-value $<10^{-5}$ as input. All other parameters were set as default.

9 Code availability

10 We made use of publicly available software and tools listed in URLs. Other codes used in

11 our analyses are available upon reasonable request.

13 Reporting summary

14 Further information on research design is available in the Nature Research Reporting 15 Summary.

16

\section{Data availability}

18 Our GWAS summary statistics have been shared at https://github.com/BIG-S2/GWAS.

19 The individual-level raw data used in this study can be obtained from five publicly 20 accessible data resources: UK Biobank (http://www.ukbiobank.ac.uk/resources/), ABCD

21 (https://abcdstudy.org/), PING (https://www.chd.ucsd.edu/research/ping-study.html),

22 PNC (https://www.med.upenn.edu/bbl/philadelphianeurodevelopmentalcohort.html),

23 and HCP (https://www.humanconnectome.org/). Our results can also be easily browsed

24 through our knowledge portal https://bigkp.web.unc.edu/. 
bioRxiv preprint doi: https://doi.org/10.1101/2020.05.23.112409; this version posted June 17, 2020. The copyright holder for this preprint a (which was not certified by peer review) is the author/funder, who has granted bioRxiv a license to display the preprint in perpetuity. It is made available under aCC-BY-NC-ND 4.0 International license.
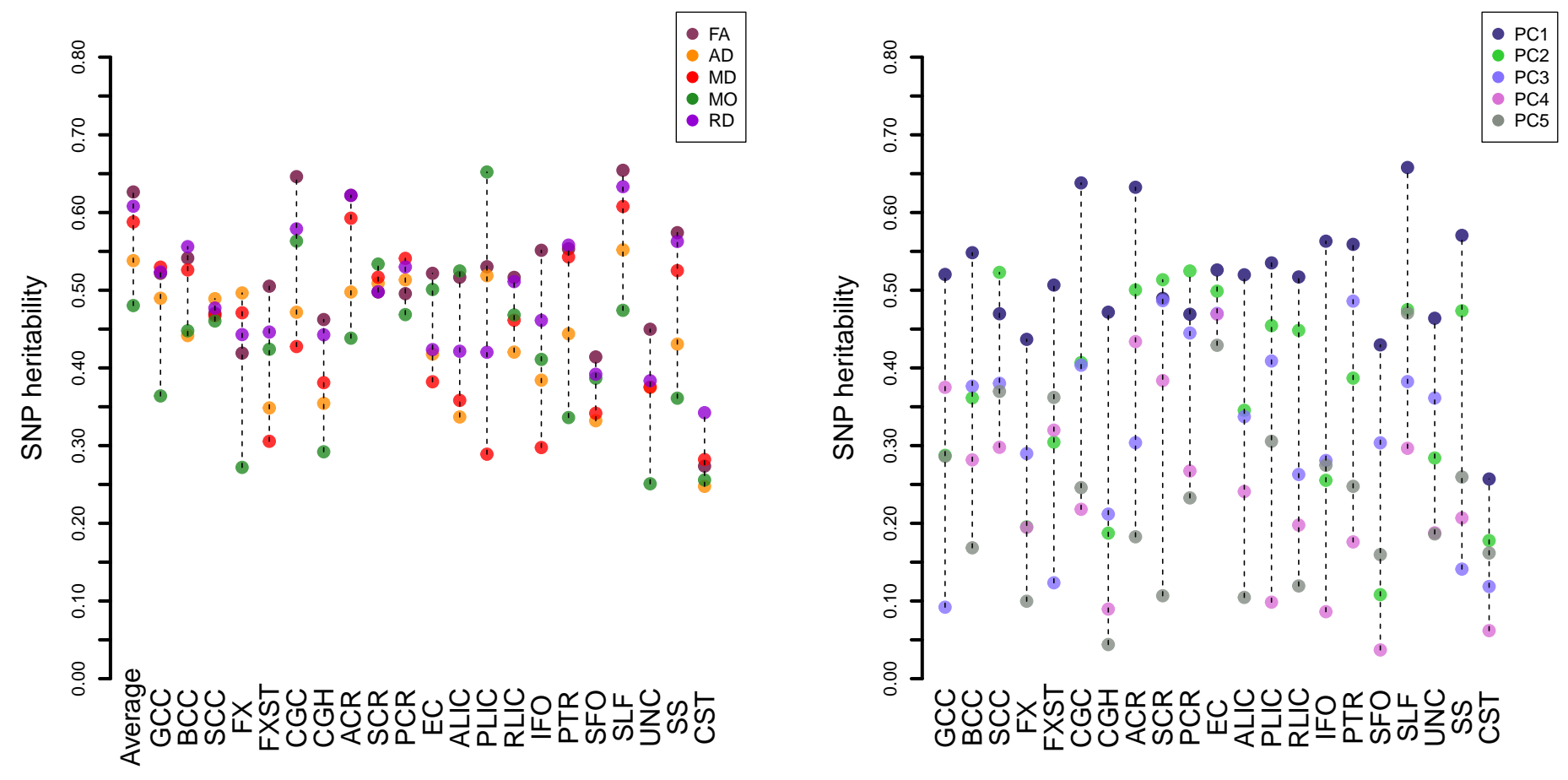

b
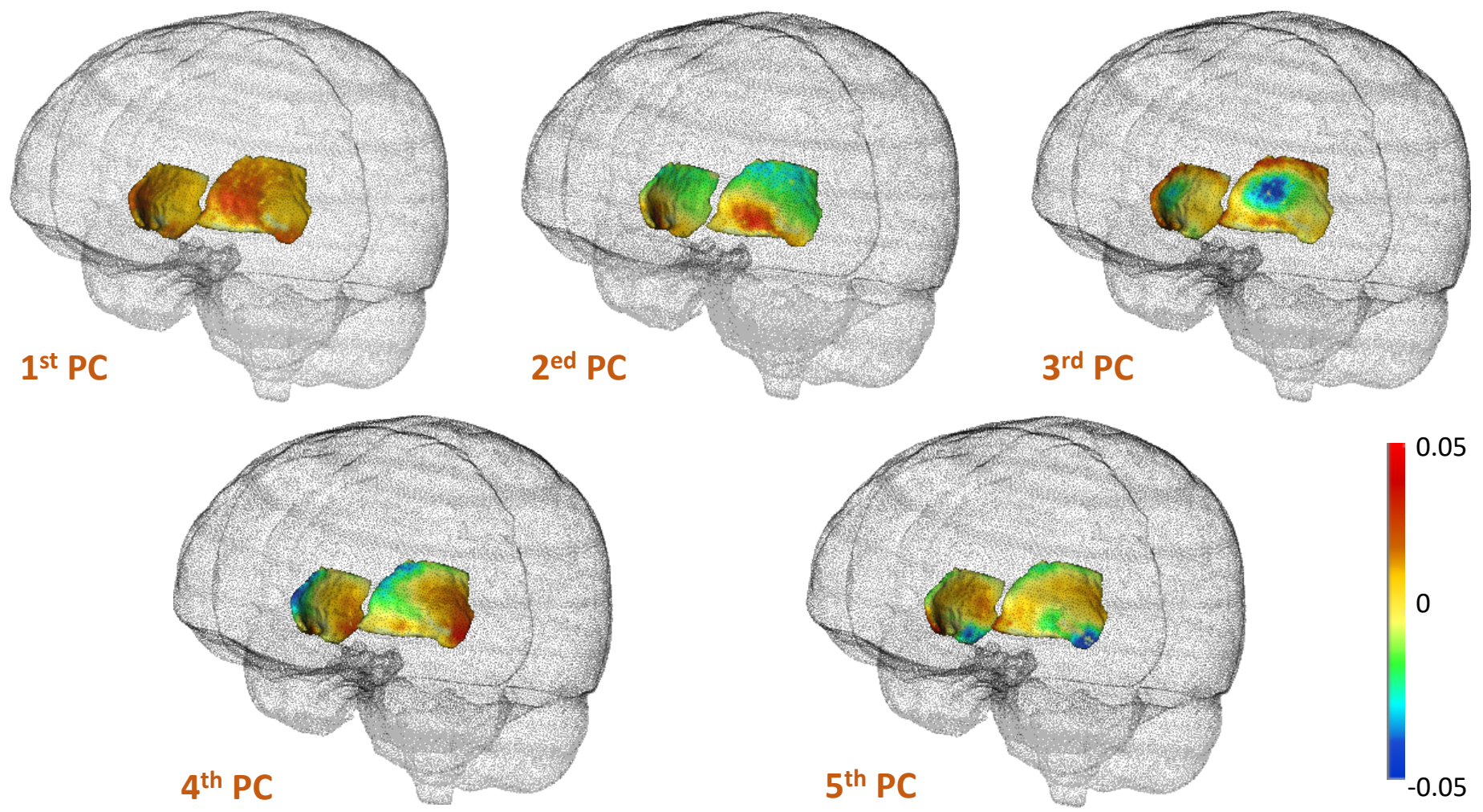

Figure 1: SNP heritability estimates of 215 DTI parameters $(\mathrm{n}=33,292$ subjects $)$ and illustration of the top five FA principal components (PCs) of external capsule (EC). a) The 110 mean DTI parameters and 105 FA PC DTI parameters are displayed on the left and right panels, respectively. The $\mathrm{x}$-axis lists the names of white matter tracts. b) The functional principal component (PC) loading coefficients for the top five FA PCs of EC. 
bioRxiv preprint doi: https://doi.org/10.1101/2020.05.23.112409; this version posted June 17, 2020. The copyright holder for this preprint (which was not certified by peer review) is the author/funder, who has granted bioRxiv a license to display the preprint in perpetuity. It is made available under aCC-BY-NC-ND 4.0 International license.

a

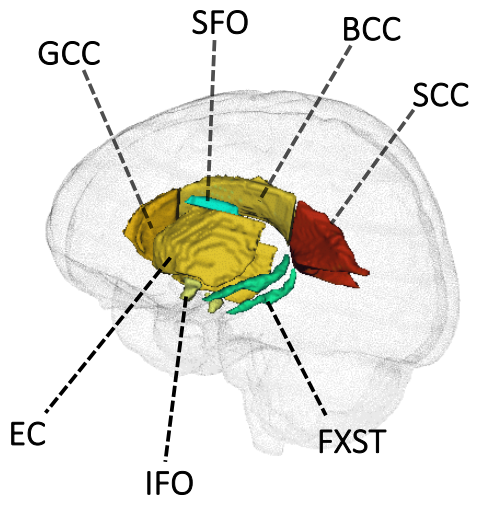

b

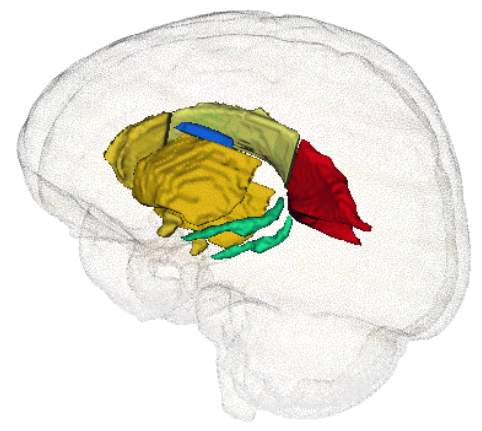

C

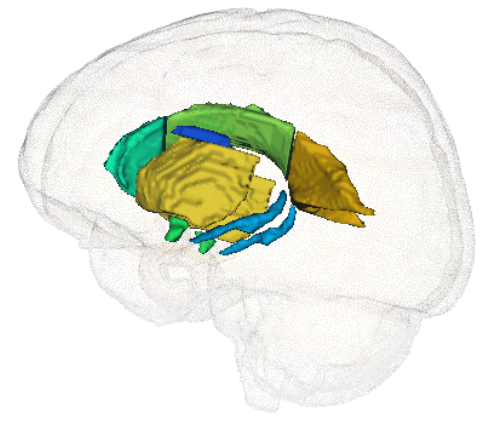

d

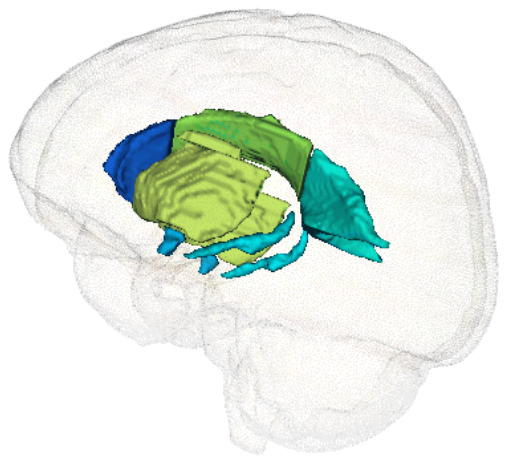

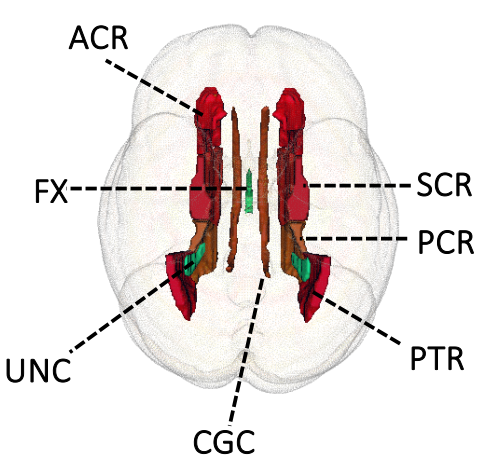
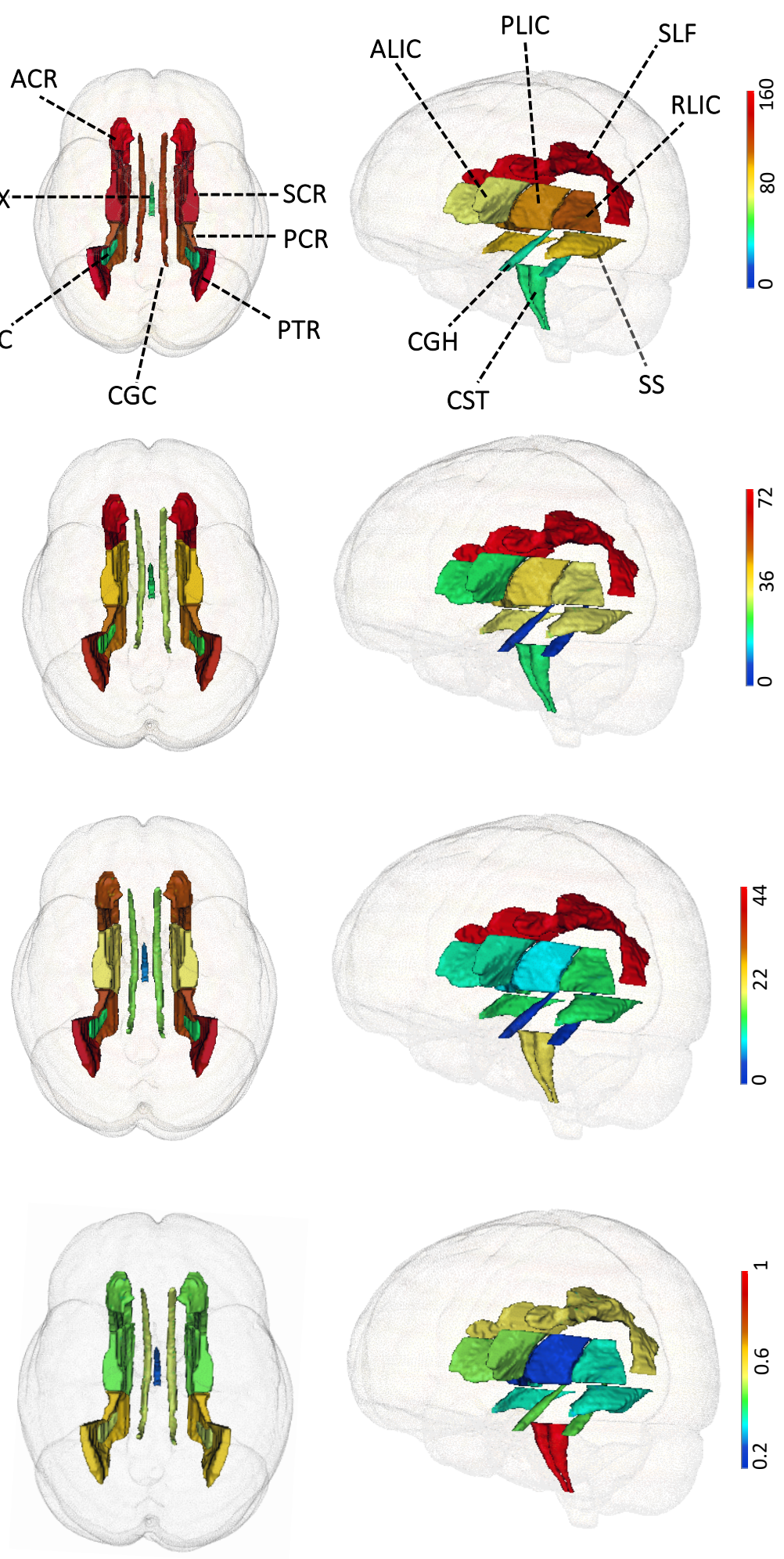

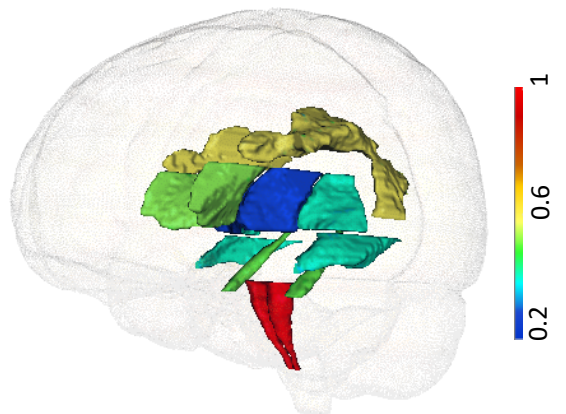

Figure 2: Number of independent significant variants identified in UKB British discovery GWAS at $2.3 \times 10^{-10}$ significance level $(\mathbf{n}=\mathbf{3 3 , 2 9 2}$ subjects $)$. The first three rows are the number of independent significant variants identified in each white matter tract by a) any DTI parameters; b) any FA parameters; c) FA PC parameters, respectively. The last row $\mathbf{d}$ ) displays the proportion of FA-associated variants that can only be identified by $\mathrm{PC}$ parameters. 
bioRxiv preprint doi: https://doi.org/10.1101/2020.05.23.112409; this version posted June 17, 2020. The copyright holder for this preprint (which was not certified by peer review) is the author/funder, who has granted bioRxiv a license to display the preprint in perpetuity. It is made available under aCC-BY-NC-ND 4.0 International license.
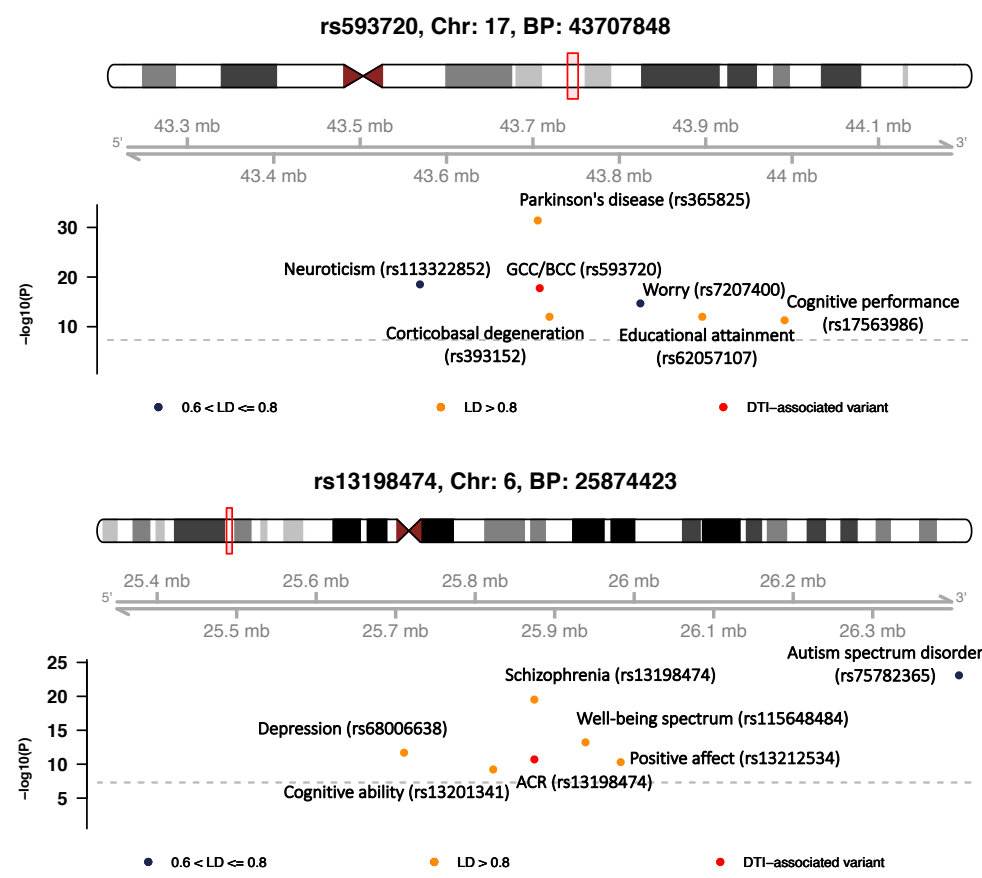

rs798510, Chr: 7, BP: 2783338

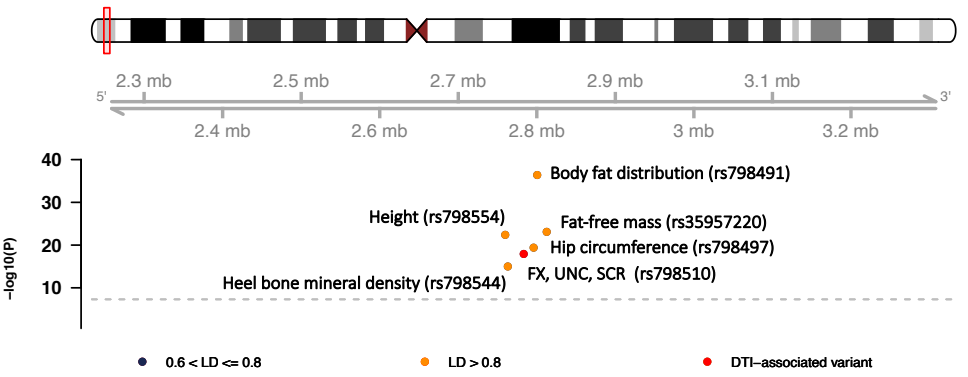

rs77126132, Chr: 7, BP: 54966738
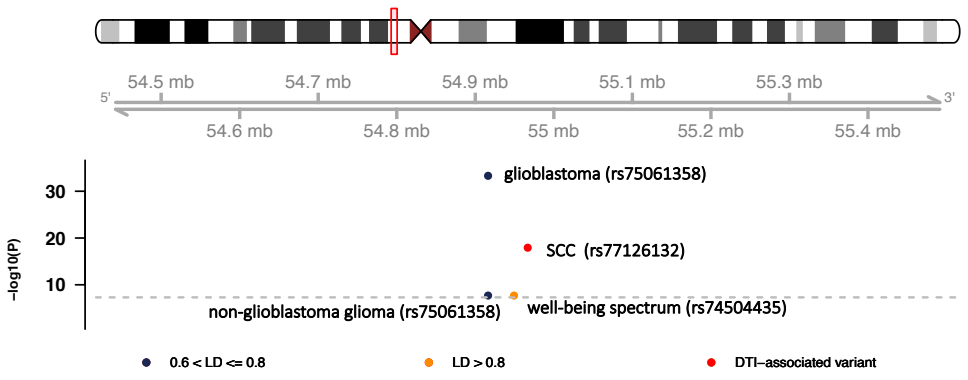

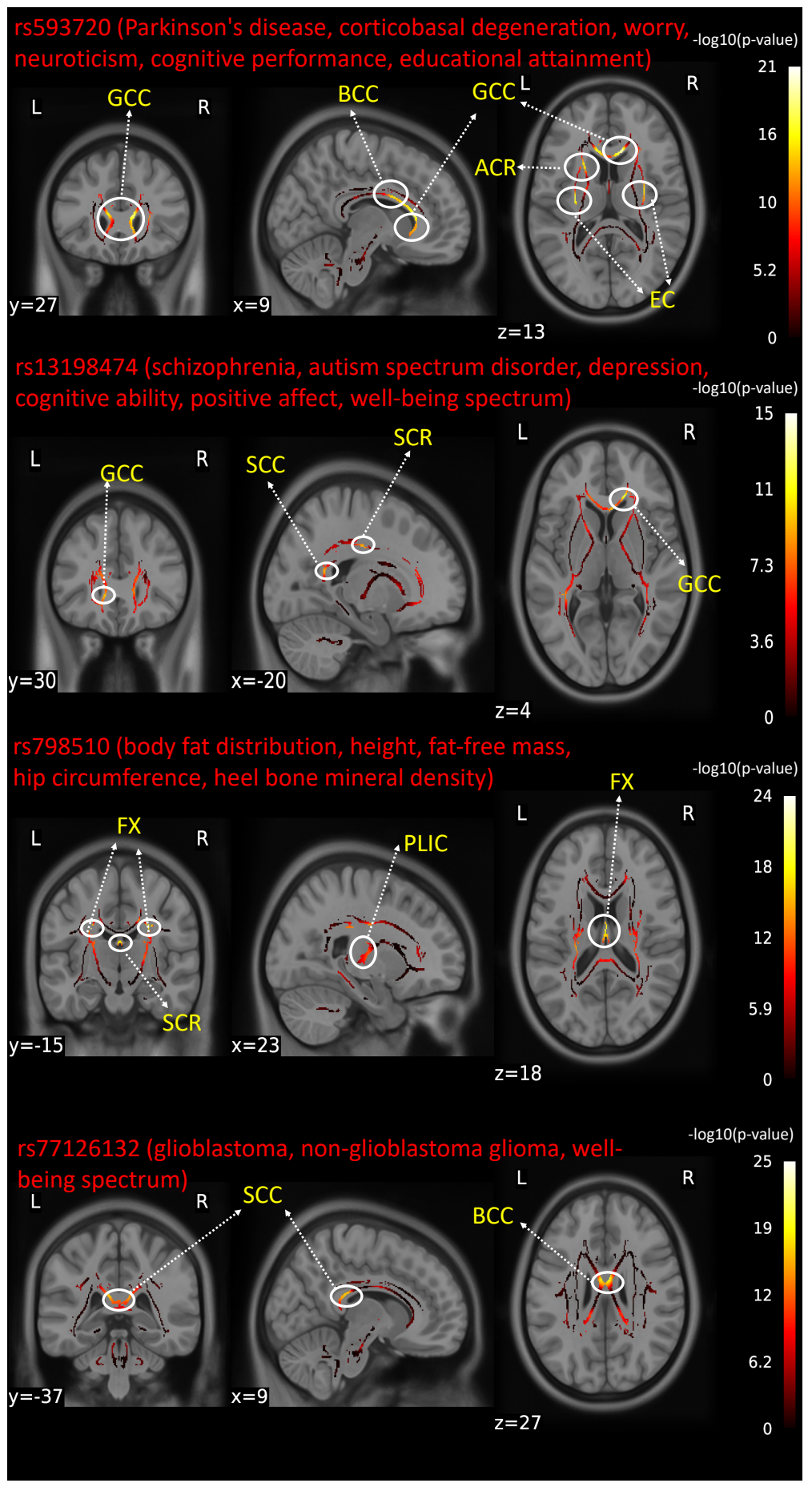

Figure 3: The genomic region and brain spatial map of voxel-wise effect size patterns for four selected pleiotropic variants $(\mathbf{n}=33,292$ subjects). We labeled previously reported GWAS variants for other complex traits in genomic regions influencing white matter microstructure (left). In spatial maps (right), we illustrate voxel-wise effect sizes of pleiotropic variants in white matter tracts. 
bioRxiv preprint doi: https://doi.org/10.1101/2020.05.23.112409; this version posted June 17, 2020. The copyright holder for this preprint (which was not certified by peer review) is the author/funder, who has granted bioRxiv a license to display the preprint in perpetuity. It is made available under aCC-BY-NC-ND 4.0 International license.
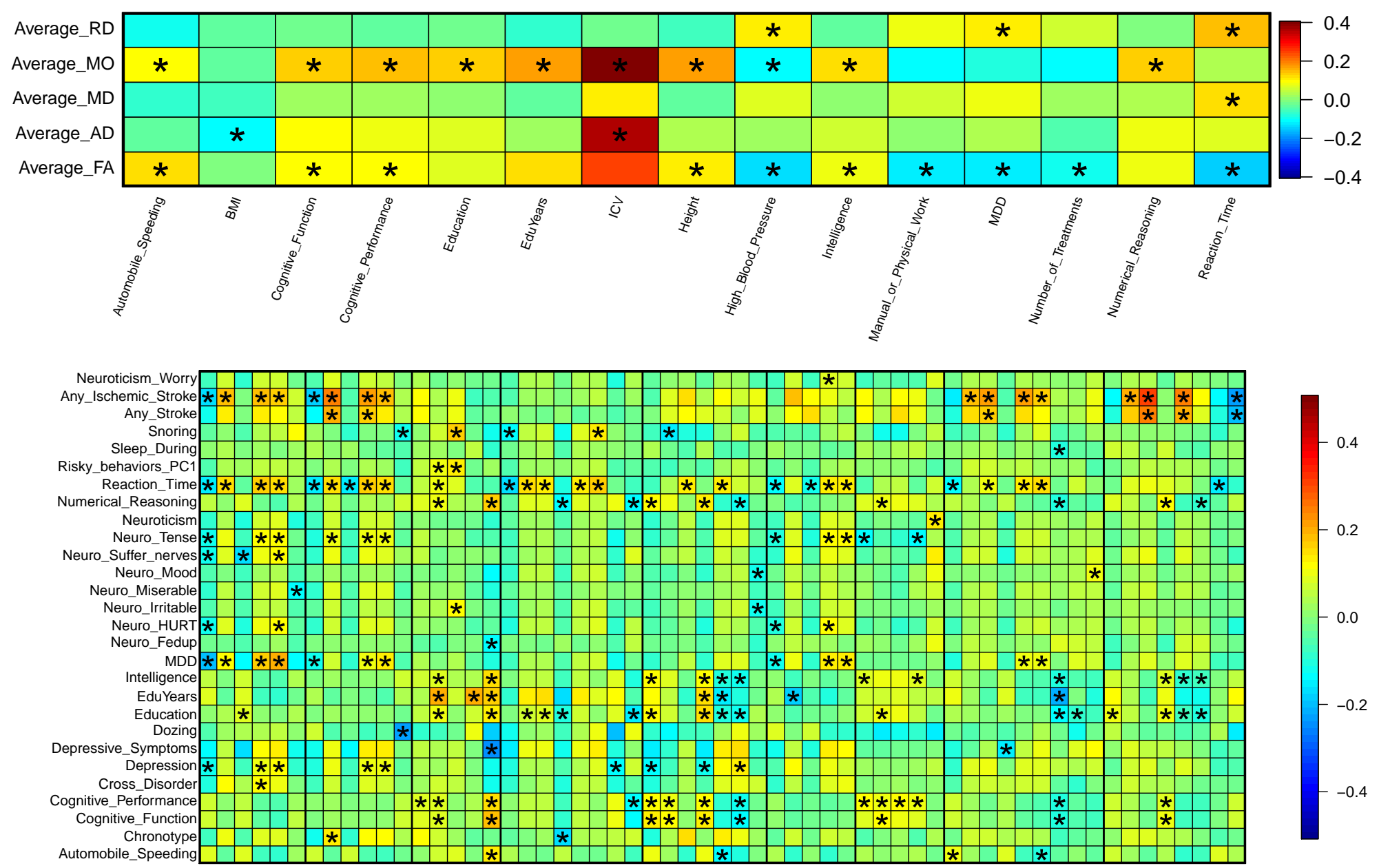

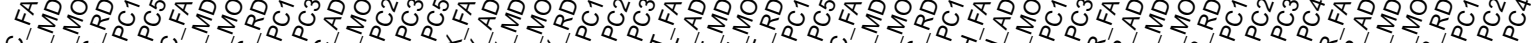

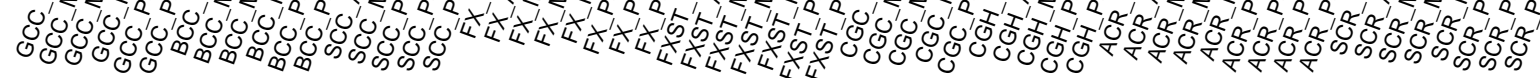

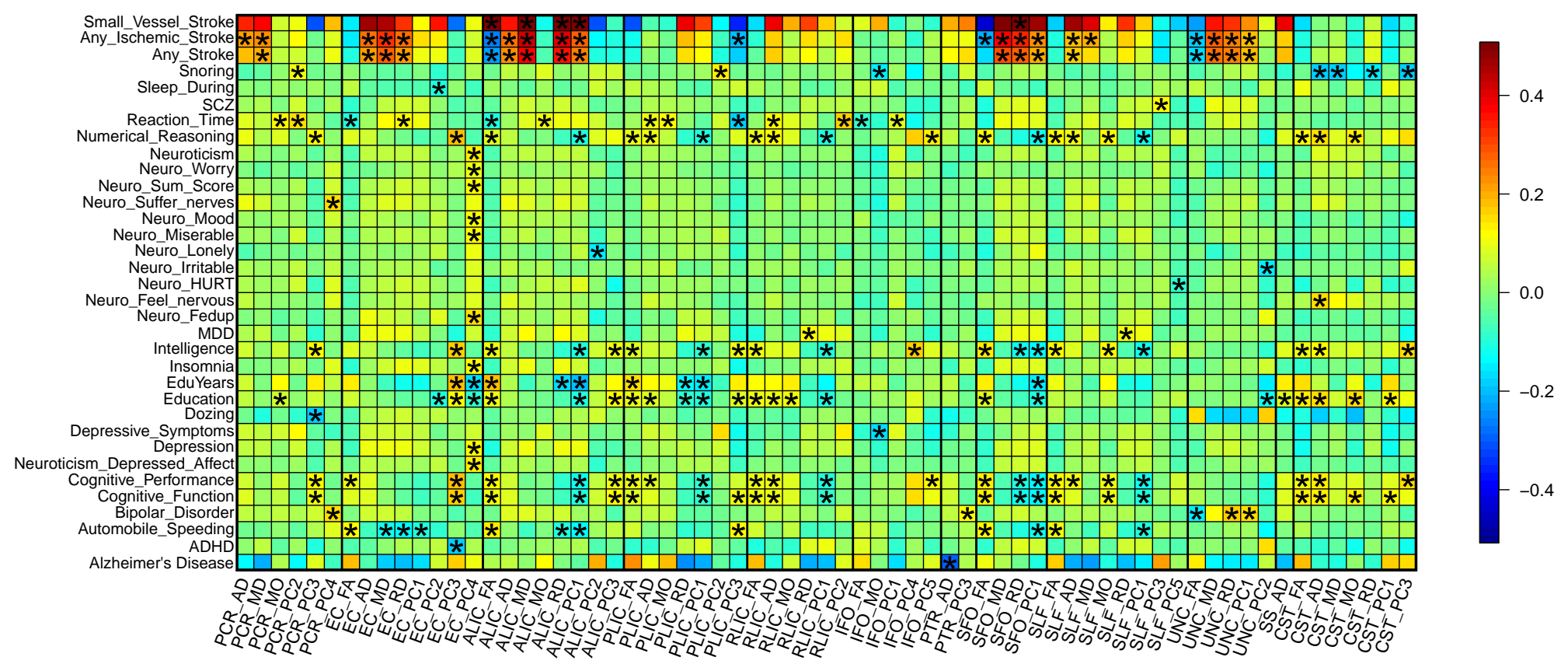

Figure 4: Selected pairwise genetic correlations between white matter microstructure and other complex traits $(\mathrm{n}=\mathbf{4 0 , 2 5 4}$ subjects). We adjusted for multiple testing by the Benjamini-Hochberg procedure at 0.05 significance level $(215 \times 76$ tests $)$, while significant pairs are labeled with stars. Sample size and detailed information of complex traits can be found in Supplementary Table 18. 

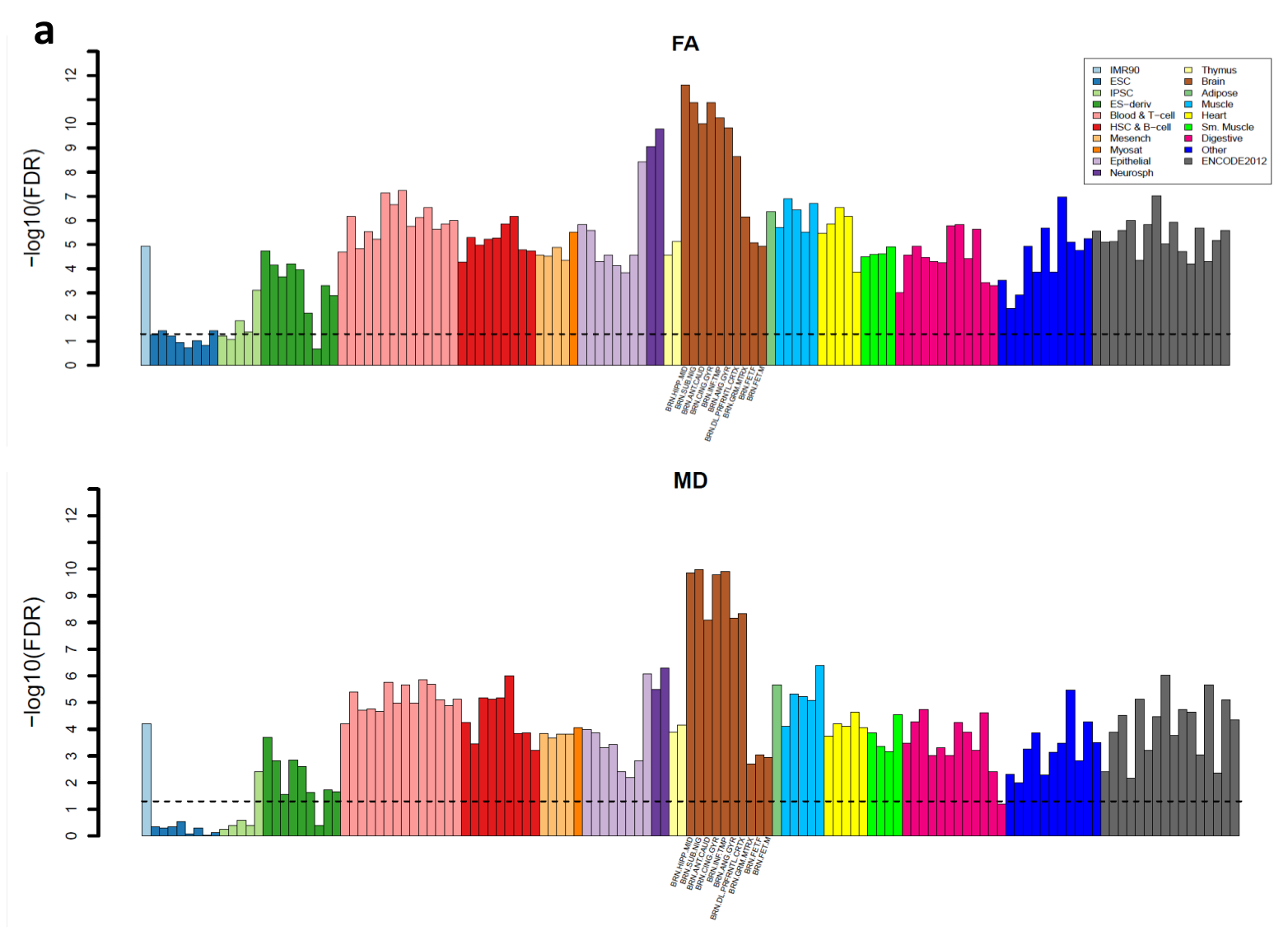

b

C
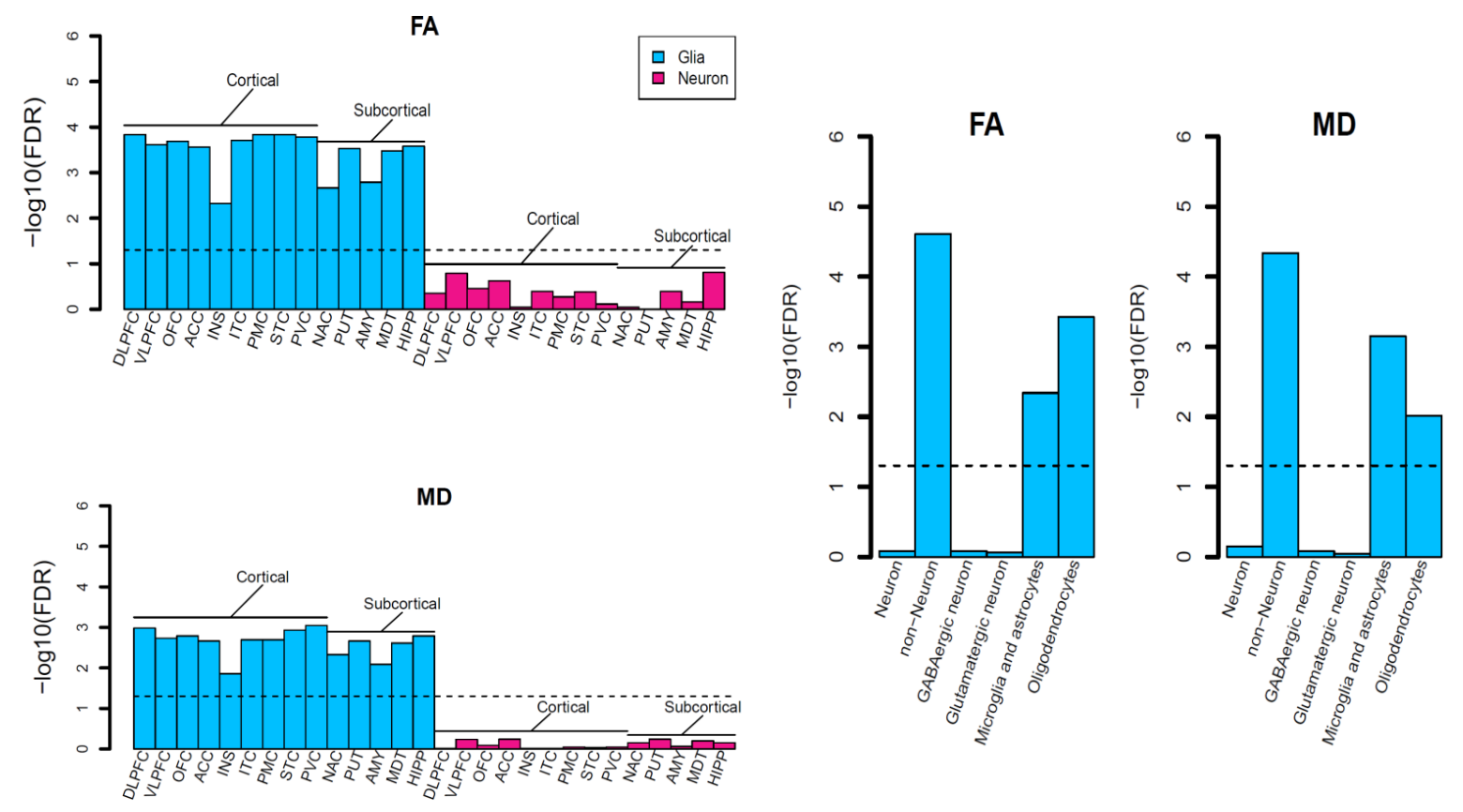

Figure 5: Partitioned heritability enrichment analysis $(\mathrm{n}=33,292$ subjects $)$. a) Heritability enrichment in regulatory elements across tissues and cell types. Brain tissues are labelled in x-axis. b) Heritability enrichment in regulatory elements of two brain cell types (neuron and glia) sampled from 14 brain regions. c) Heritability enrichment in regulatory elements of glial cell subtypes (non-neuron, including oligodendrocyte and microglia \& astrocyte) and neuronal cell subtypes (neuron, including GABAergic and glutamatergic neurons). 